\title{
A Surrogate Model Approach in 2D versus 3D Flapping Wing Aerodynamic Analysis
}

\author{
Pat Trizila ${ }^{1}$ and Chang-kwon Kang ${ }^{1}$ \\ Department of Aerospace Engineering, University of Michigan, Ann Arbor, Michigan 48109 \\ Miguel Visbal ${ }^{2}$ \\ U.S. Air Force Research Laboratory, Wright-Patterson Air Force Base, Ohio 45433 \\ and \\ Wei Shyy ${ }^{3}$ \\ Department of Aerospace Engineering, University of Michigan, Ann Arbor, Michigan 48109
}

\begin{abstract}
Flapping wing MAV aerodynamics is challenging to understand due to its complexities. Surrogate modeling offers an effective tool to predict information at off-design points, measure the sensitivity of design variables involved, and illustrate general trends in the data. We have investigated 2D and 3D hovering airfoil/wing aerodynamics considering three kinematic parameters, i.e. plunge amplitude, angular amplitude, and phase lag, at $\operatorname{Re}=100$. While leading edge vortex, a persistent downward jet, and wake capture are three noticeable fluid dynamics features in 2D and 3D, tip vortices in 3D and instantaneous AoA can substantially affect the relative importance of them. Surrogate models of the 2D and 3D wing with aspect ratio of 4 show that for i) power required, the magnitudes and global trends of the predicted response between $2 \mathrm{D}$ and $3 \mathrm{D}$ are similar, ii) lift, the phase lag changes from a non-linear response in 2D to a monotonic one in 3D. The overall consequence is that in 3D, kinematic combinations of higher lift with lower power requirements can be attained. Global sensitivity analysis shows that the lift is the most sensitive to the phase lag in 3D as opposed to the angular amplitude in $2 \mathrm{D}$. The ensemble surrogate strategies performed employed performed quite well compared to independent test data.
\end{abstract}

$\begin{array}{lll} & & \\ \mathrm{AR} & = & \text { aspect ratio } \mathrm{b}^{2} /(\mathrm{bc}) \\ \mathrm{b} & = & \text { Nomenclature } \\ \mathrm{C}_{\mathrm{D}} & = & \text { drag coefficient } \\ \mathrm{C}_{\mathrm{L}} & = & \text { lift coefficient } \\ \mathrm{c} & = & \text { chord length } \\ \mathrm{DOE} & = & \text { design of experiment } \\ \mathrm{f} & = & \text { frequency of oscillation } \\ \mathrm{FCCD} & = & \text { face centered cubic design } \\ \mathrm{GCL} & = & \text { geometric conservation law } \\ \mathrm{GSA} & = & \text { global sensitivity analysis } \\ \mathrm{h} & = & \text { translational position } \\ \mathrm{h}_{\mathrm{a}} & = & \text { plunging amplitude } \\ \mathrm{k} & = & \text { reduced frequency } \\ \mathrm{KRG} & = & \text { Kriging }\end{array}$

\footnotetext{
${ }^{1}$ Graduate Student, Department of Aerospace Engineering, University of Michigan, Member AIAA.

${ }^{2}$ Technical Area Leader, Computational Sciences Branch, AFRL/RBAC, Associate Fellow AIAA.

${ }^{3}$ Clarence L. "Kelly" Johnson Collegiate Professor and Chair, Department of Aerospace Engineering, University of Michigan, Fellow AIAA.
} 


\begin{tabular}{|c|c|c|}
\hline LEV & $=$ & leading edge vortex \\
\hline LHS & $=$ & Latin hypercube sampling \\
\hline $\mathrm{L}_{\mathrm{ref}}$ & $=$ & reference length \\
\hline MAV & $=$ & micro air vehicle \\
\hline $\mathrm{N}_{\mathrm{DV}}$ & $=$ & number of design variables \\
\hline $\mathrm{N}_{\mathrm{SM}}$ & $=$ & number of surrogate models \\
\hline $\mathrm{N}_{\mathrm{TP}}$ & $=$ & number of training points \\
\hline $\mathrm{p}$ & $=$ & pressure \\
\hline$P_{\text {req }}$ & $=$ & power required \\
\hline PRESS & $=$ & prediction error sum of squares \\
\hline PRS & $=$ & polynomial response surface \\
\hline Q & & second invariant of velocity gradient tensor \\
\hline $\operatorname{Re}$ & $=$ & Reynolds number \\
\hline RBNN & $=$ & radial basis neural network \\
\hline $\mathrm{S}_{\mathrm{ij}}$ & $=$ & rate-of-strain tensor \\
\hline SVR & $=$ & support vector regression \\
\hline $\mathrm{T}$ & $=$ & period of oscillation \\
\hline $\mathrm{t}$ & $=$ & time \\
\hline $\mathrm{u}$ & $=$ & velocity \\
\hline $\mathrm{U}_{\max }$ & $=$ & maximum velocity \\
\hline $\mathrm{U}_{\text {ref }}$ & $=$ & reference velocity \\
\hline WAS & $=$ & weighted average surrogate \\
\hline $\mathrm{X}_{\mathrm{i}}$ & $=$ & the ith design variable \\
\hline $\mathbf{x}$ & $=$ & the vector of $\mathrm{N}_{\mathrm{DV}}$ design variables $\left\{\mathrm{x}_{1}, \mathrm{x}_{2}, \ldots, \mathrm{x}_{\mathrm{NDV}}\right\}$ \\
\hline $\mathbf{X}$ & $=$ & Gramian design matrix \\
\hline $\mathbf{X}^{\prime}$ & $=$ & $\mathbf{X}$ transpose \\
\hline $\mathrm{y}$ & $=$ & actual individual response from physical model \\
\hline $\mathbf{y}$ & $=$ & vector of solutions \\
\hline$\hat{y}$ & $=$ & predicted response by the surrogate model \\
\hline$\alpha$ & $=$ & angular position \\
\hline$\alpha_{0}$ & $=$ & initial angular position \\
\hline$\alpha_{\mathrm{a}}$ & $=$ & angular amplitude \\
\hline$v$ & $=$ & kinematic viscosity \\
\hline$\rho$ & $=$ & density \\
\hline$\varphi$ & $=$ & phase lag \\
\hline$\sigma$ & $=$ & standard deviation \\
\hline$\omega_{\mathrm{i}}$ & $=$ & vorticity vector \\
\hline$<x>$ & $=$ & time averaged value of some quantity $\mathrm{x}$ \\
\hline
\end{tabular}

\section{Introduction}

M icro air vehicles (MAVs) with a maximal dimension of $15 \mathrm{~cm}$ or less and a flight speed of $10 \mathrm{~m} / \mathrm{s}$ are interests of both military and civilian applications. Equipped with a video camera or a sensor, these vehicles can perform surveillance and reconnaissance, and environmental and bio-chemical sensing at remote or otherwise hazardous locations. In addition, from the scaling laws ${ }^{1}$, a MAV's payload is very limited; sensors, batteries, communications equipment, and means of sustained propulsion will have to compete for precious cargo space only in as much as that they will directly help defined mission statements. Research regarding MAVs is growing as there are still many open challenges in theory and in practice.

There are three different design approaches in MAV development. (i) The fixed wing vehicles ${ }^{2}$ resemble miniaturized versions of conventional fixed wing aircraft except that flexible wings are often utilized. (ii) Small rotor based ${ }^{3}$ vehicles resembling helicopters are also pursued, where often multiple rotors are used. One defining characteristic for which these vehicles are valued is their ability to hover, allowing for monitoring systems which can remain stationary or follow targets as the situation dictates. (iii) Of interest in the current study are the flapping wing variety ${ }^{4}$ of MAVs which take a cue from nature and attempt to mimic the success achieved by insects, birds, and bats, see Refs. 5-7. Common to all three classifications is the sensitivity to perturbations in the environment 
such as wind gusts, assessing the impact of transition to turbulence which due to the scales involved may or may not be relevant and can be difficult to gauge a priori, the need for coupled fluid-structure interaction capabilities to capture the changes in the vehicle induced by the flow, the advanced control schemes needed to successfully fly these vehicles autonomously, and the understanding of the unsteady flow mechanisms present and their respective roles in the aerodynamic loadings on the MAV.

The study of flapping wing flyers with all of their intricacies is challenging. However, as summarized in Refs.1,4, and 8-16 significant progress has been made in both engineering and biological communities. Computational studies of realistic wing body configurations of a hornet ${ }^{17}$, hawkmoth ${ }^{18}$, fly, and thrip ${ }^{1,19,20}$ accompanied by appropriate wing kinematics demonstrate that such feats are within reach. A few potential difficulties encountered are due to the desired complexity of the setup; meaningful analysis requires a more sophisticated approach and the simulations themselves are computationally expensive. On the experimental side, characteristics of insects and animals in their natural habitat are understandingly hard to quantify. High speed photography and PIV measurements are beginning to offer data for detailed assessment ${ }^{21,22}$. A few groups have taken these natural flyers and not only introduced them to environments more accommodating of detailed study, but also trained and/or manipulated the flyer to perform desired behaviors ${ }^{23}$. Smoke tunnel visualizations of a hawkmoth ${ }^{24}$ demonstrated the relevance of unsteady flow structures. Detailed measurements of fruitflys ${ }^{25}$, hawkmoths ${ }^{26}$, butterflys ${ }^{27}$, dragonflys ${ }^{28}$, bats ${ }^{29}$, hummingbirds ${ }^{21,30}$, and swifts ${ }^{31}$ in flight have resulted in realistic wing kinematic descriptions, force estimates, and flowfield descriptions in ever increasing precision.

In order to improve our understanding of the fundamentals of flapping wing aerodynamics, it is valuable to simplify the problem to illuminate specific aspects in greater detail. Experimental investigations regarding the shape of dragonfly wings ${ }^{32,33}$ have examined the effect of small vortical flow features captured by a corrugated flat plate on the overall aerodynamic performance at low Reynolds numbers. Kinematic studies of flapping wings often reduce the number of possible design variables by looking at representative motions that serve a specific purpose such as forward flight, maneuvering, or hovering. Representative hovering kinematics have been used in a number of studies to gain a better understanding of the unsteady flow physics involved in flapping flight. Ref. 34 looks at the impact of the interacting aerodynamics of a $2 \mathrm{D}$ fore-wing hind-wing combination and concludes that those influences are indeed a dominant factor in the aerodynamic performance. Ref. 35 compares 2D computational simulations of an ellipse against experimental results. PIV measurements are found in Ref. 36, detailing experimental observations of flapping wing flight with hovering kinematics. Two dimensional computational studies are examined in Ref. 37 of different hovering kinematics found in the literature while Ref. 38 gauges the sensitivity of the kinematic parameters by tying the consequences to the resulting unsteady mechanisms and finally to the aerodynamic loadings. Ref. 39 carries out a comprehensive experimental study of five kinematic hovering variables and compared their results to the quasi-steady predictions. While the mean lift value over the course of a wing cycle matched reasonably, the instantaneous values differed significantly. The implication here is that a potential control device, for which moments and therefore instantaneous forces are important, would not be able to use quasi-steady approximations as a feasible reduced order model to be used in real time calculations. They also saw that the mean drag was greatly underestimated which would in turn under predict power required estimates.

Various unsteady lift enhancing mechanisms help enhance the instantaneous steady state lift values during hovering ${ }^{1}$. Delayed stall is one such mechanism that comes about as a leading edge vortex ${ }^{24}$ (LEV) forms on the upper surface of the airfoil. This in turn causes a low pressure region on the upper surface which increases the lift. Wake capture refers to when the airfoil switches direction and encounters the previously shed wake, which can then be taken advantage of if at the proper orientation. Weis-Fogh's clap-and-fling ${ }^{40}$ takes advantage of two wings coming together and subsequently peeling away from one another to enhance performance. Other unsteady features, such as jet-interaction $^{38}$, tend to decrease performance. Because of the complex relationships between the kinematic motions and the resulting aerodynamic loading, the general trends are not easily discernable. Many simulations can be run and even then the known data are only a minute fraction of the possible combinations. Each of these simulations can take hundreds and even thousands of computational hours, and while parallel computing makes the runs realistic, computing resources are finite. This is where use of surrogate modeling becomes desirable.

Surrogate models are a valuable and versatile tool in engineering design. Known data are typically limited, and in disciplines requiring substantial experimental setups or costly computational simulations, inquiring about off design points may not yield satisfactory answers. Strengths of the surrogate modeling methodology are the ability to provide information at off-design points and do so rather quickly once the requisite training information has been obtained. This in turn allows one to obtain measures of a variable's relevance via its sensitivity, an important determination in any design process. While one of the drawbacks of the surrogate modeling approach is that the training step can be expensive, if one can glean enough information from another source one can reduce and maybe even bypass this step (e.g., estimating data points from an experimental study in a journal) or perform it in parallel. 
An overview of surrogate modeling techniques detailing the process and many of the available options can be found in Ref. 41. Popular models are polynomial response surfaces (PRS) ${ }^{42}$, Kriging ${ }^{43}$, radial basis neural networks $(\mathrm{RBNN})^{44}$, support vector regression (SVR $)^{45}$ models and various combinations thereof. Because of the surrogate models' ability to provide quick reduced order approximations, i.e. orders of magnitude faster than a CFD simulation, they become an attractive tool in engineering design ${ }^{46}$ and optimization ${ }^{47,48}$. Engineering applications looking for optimal settings include vibration reduction in helicopter flight ${ }^{49}$, flap and slat configurations for airfoils in turbulent flow $^{50}$, and shape optimization in various contexts ${ }^{51-54}$. The surrogate modeling process is more generally applicable however as seen by the uses to find robust solutions ${ }^{55}$ (i.e. because manufacturing uncertainties will introduce deviations from the design point) and calibration ${ }^{56}$ of numerical models against experiment. Recently these tools have been used as the primary means of exploring the behavior behind physical interactions involved with plasma actuators ${ }^{57}$, Lithium-Ion battery limitations ${ }^{58}$, and flapping wing micro air vehicle (MAV) flight ${ }^{20,38}$.

Guaranteeing the quality of surrogate models is not automatic. Some models, e.g., PRS and Kriging, have error predictions available due to the way in which they were constructed ${ }^{59}$. However not all models share this characteristic. One global error metric that can be evaluated for any choice of surrogate after the models are built is the prediction error sum of squares (PRESS) ${ }^{60}$. Non-acceptable PRESS values can immediately identify bad surrogate models. One cannot immediately conclude a good PRESS value is a good surrogate, as it may just happen to model the training data especially well without being able perform equally well in the rest of the domain. Two methodologies $^{60,61}$ currently employed weight the surrogate model candidates, those with good PRESS values, to form a combination which mitigates the risk of obtaining a bad surrogate with good PRESS characteristics.

To improve our understanding of the aerodynamics and the underlining fluid physics related to flapping wing MAVs, we have previously utilized the composite surrogate modeling techniques to probe the aerodynamic implications of a $2 \mathrm{D}$ flapping airfoil ${ }^{38}$. Addressed in that study is the impact of kinematic parameters and Reynolds number (100-1000) under free stream/hovering conditions. The kinematic parameters include plunging amplitude, angular amplitude, and pitching/plunging phase angle. With the aid of the global sensitivity analysis, the individual and collective influence of the kinematic parameters (also referred to as design variables) can be ranked. In the current study, we assess both 2D and 3D flapping wing fluid physics at Reynolds number of 100, and the impact of the above-mentioned kinematic variables on the aerodynamic outcome for representative hovering wing motions. Regarding the surrogate modeling techniques, the implications of the weighting strategies to obtain the ensemble surrogates are also addressed.

\section{Approaches and Tools}

\section{A. Computational Models}

The governing equations are the laminar, unsteady, Navier-Stokes equations with constant transport properties; the incompressible versions are shown in Eqs. (1) and (2) written in indicial form.

$$
\begin{gathered}
\frac{\partial}{\partial x_{j}}\left(u_{j}\right)=0 \\
\frac{\partial}{\partial t}\left(u_{i}\right)+\frac{\partial}{\partial x_{j}}\left(u_{j} u_{i}\right)=-\frac{1}{\rho} \frac{\partial p}{\partial x_{i}}+v \frac{\partial}{\partial \mathrm{x}_{j}}\left(\frac{\partial u_{i}}{\partial x_{j}}\right)
\end{gathered}
$$

Here $u_{i}$ is the velocity component in the $\mathrm{i}^{\text {th }}$ direction, $x_{i}$ is the $\mathrm{i}^{\text {th }}$ Cartesian position vector, $t$ is time, $\rho$ is density, $p$ is pressure, and $v$ is the kinematic viscosity. A rule-based software ${ }^{62}$, Loci-STREAM ${ }^{63}$ is used to calculate the solutions. Loci-STREAM is a three-dimensional, unstructured, pressure based solver. The present calculations use implicit first or second order time stepping (the first order technique is adopted in this study). The convection terms are treated using the second order upwind scheme ${ }^{64,65}$ while pressure and viscous terms are treated using second order central differencing schemes. The system of equations resulting from the linearized momentum equations are fast to converge ${ }^{65}$ and are handled with the symmetric Gauss-Seidel ${ }^{66}$ solver which has relatively low memory requirements. The pressure equation, derived in Refs. 63 and 67, is slower to converge, and is handled by the PETSc Krylov $^{66}$ solvers with Jacobi preconditioning. The Loci framework is by design highly parallelizable and can take advantage of many processors.

The translational and rotational airfoil/grid motions are dictated by Eqs. (3) and (4). 


$$
\begin{gathered}
h(t)=h_{a} \sin (2 \pi f t) \\
\alpha(t)=\alpha_{0}-\alpha_{a} \sin (2 \pi f t+\phi)
\end{gathered}
$$

Here $h(t)$ and $h_{a}$ are the translational position and plunging amplitude respectively. The angular orientation, initial angle, and angular amplitude are $\alpha(t), \alpha_{0}$, and $\alpha_{a}$ respectively. The pitching is about the center of the rigid airfoil; this is an ellipse having a 15\% thickness for all cases under consideration. The phase lag between the two motions is $\phi$, and the frequency is denoted $f$ whereas the time is again $t$. While there are a few choices in how to accommodate these kinematics computationally, the current implementation forces the grid to rotate and translate with airfoil. The geometric conservation law $^{68}$ (GCL), a necessary consideration in domains with moving boundaries, is satisfied ${ }^{69}$. The boundary condition applied to all outer boundaries is the incompressible inlet with density and velocity specified. The three quantities that we can independently vary are $h_{a}, \alpha_{a}$, and $\phi$.

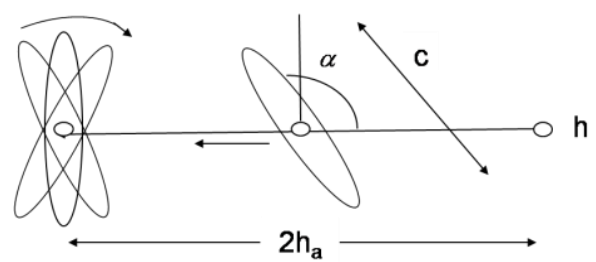

Figure 1. Illustration of the kinematic parameters for normal hovering.

Due to the kinematic constraints there are only two relevant non-dimensional groups in the incompressible case. The plunging amplitude to chord ratio, $2 h_{a} / c$, and the Reynolds number:

$$
R e=\frac{U_{r e f} L_{r e f}}{v}
$$

Rewritten for hovering the $R e$ becomes:

$$
R e_{\text {hovering }}=\frac{\left(2 \pi f h_{a}\right) c}{v}
$$

The reference velocity in this case is the maximum translational velocity, defined by the flapping frequency, $f$, and the plunging amplitude, $h_{a}$. Since $R e$ is being held constant, $h_{a}$ and $f$ are not independent. Note that the reduced frequency, $k$, is not emphasized here as, in the absence of a freestream, it contains the same information as the plunging amplitude ratio whereas if $U_{\text {ref }}$ is instead set equal to zero the reduced frequency is always infinite.

$$
k_{\text {hovering }}=\frac{2 \pi L_{r e f}}{2 U_{r e f}}=\frac{2 \pi f c}{2\left(2 \pi f h_{a}\right)}=\frac{c}{2 h_{a}}
$$

Figure 2 shows the grid distribution near the flat plate, and the applied boundary conditions over the computational domain. The thickness of the flat plate is $0.02 c$, and the flat plate is rectangular, i.e. there is no variation in spanwise direction (z-axis). The half-span length from the symmetry plane to the wingtip is $2 c$, the leading, and trailing edges are rounded using a half circular shape, while the wingtip is flat. The outer boundary is located at $25 c$ away from the flat plate, and the outer boundary plane opposite to the symmetry plane at $15 c$. At all outer boundaries the zero velocity conditions are imposed. 

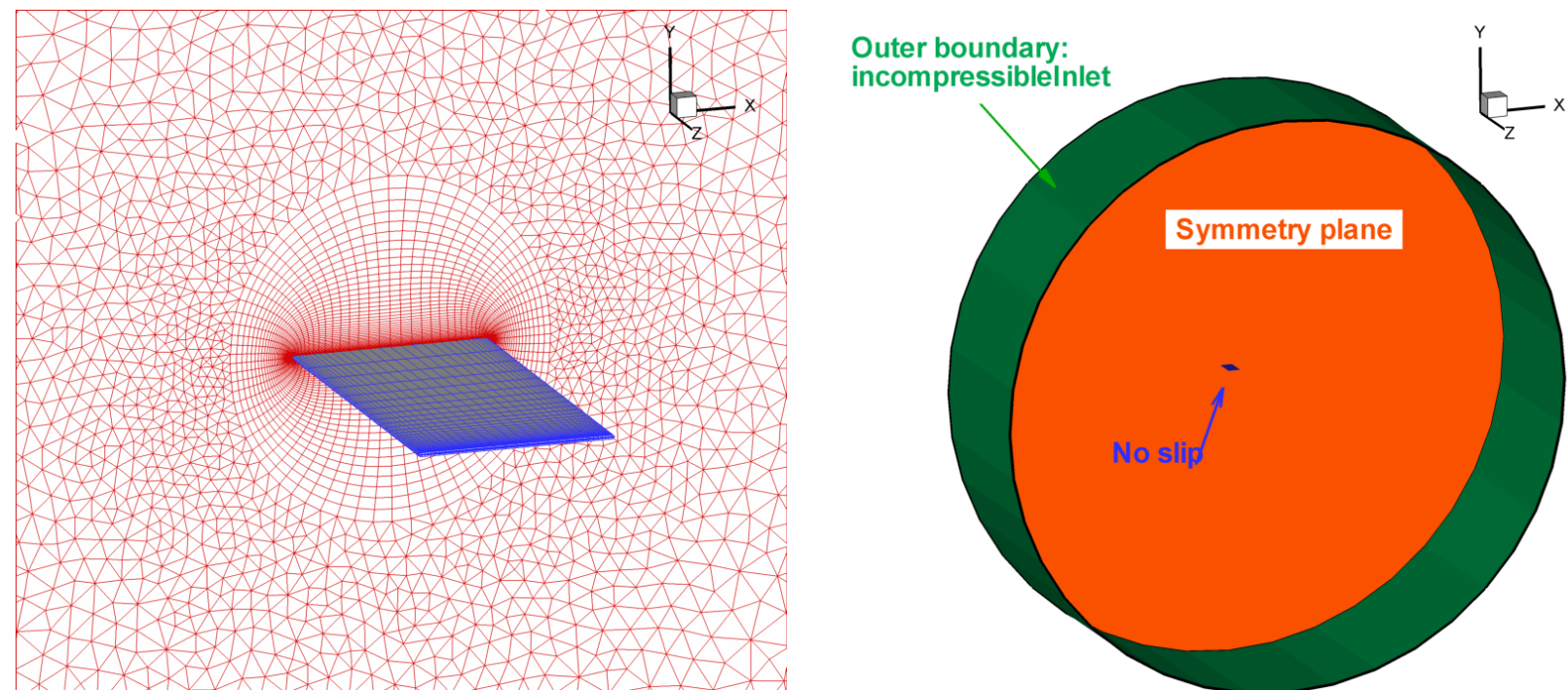

Figure 2. (Left): Grid distribution on the flat plate, and on the symmetry plane. (Right): Boundary conditions assigned on the computational domain. The outer boundary plane opposite to the symmetry plane has not been shown due to visibility also has the incompressibleInlet boundary condition.

The 2D surrogate modeling cases use a $15 \%$ ellipse while the 3D cases use a $2 \%$ thick flat plate with an AR=4. Grid and temporal sensitivity studies are included in the appendix as are the results of a cross-validation exercise. Select cases were simulated with a 2D flat plate, to separate the 3D flow physics from the shape impact and are shown in the results section. For these $2 \mathrm{D}$ flat plate cases the symmetry plane in the $3 \mathrm{D}$ flat plate grid is used as the computational domain.

\section{B. Surrogate Modeling}

The motivation behind surrogate models is to replace costly objective function evaluations with inexpensive approximations of sufficient fidelity. The process starts with choosing how many training points are needed and then specifying those points; this is known as constructing the design of experiment (DOE). Once the training points are chosen, the objective functions must be evaluated; depending on the context this can be done computationally or experimentally. After the appropriate objective functions have been obtained for the selected training points, the surrogate models can be built. After the models are constructed, the PRESS error metric can be evaluated to obtain the candidate models for the ensemble surrogate. Finally, once weighted average surrogate (WAS) is obtained, the global sensitivity evaluations can be performed.

\section{Design Space}

The range of variables, see Table 1, was chosen after considering the length and time scales observed in nature and compiled in Refs. 40, 70, and 71, see Table 2. Tabulated measurements of angular amplitudes and phase lags for a variety of species are not as forthcoming, however the comments in those references would indicate that bounds chosen are reasonable. As a first attempt, we focus on the Reynolds number of 100 under hovering conditions for which representative values have been calculated, see Table 2. For the 3D cases, the aspect ratio of 4 was chosen. Both elliptic airfoil and flat plate have been considered in 2D cases. The flat plate is exclusively used in 3D cases.

\begin{tabular}{|l|c|c|}
\hline Parameter & Minimum & Maximum \\
\hline $2 h_{d} / c$ & 2.0 & 4.0 \\
\hline$\alpha_{a}$ & $45^{\circ}$ & $80^{\circ}$ \\
\hline$\varphi$ & $60^{\circ}$ & $120^{\circ}$ \\
\hline
\end{tabular}

Table 1. Minimum and maximum values of the plunging amplitude ratio, angular amplitude, and phase lag that were evaluated. 


\begin{tabular}{|l|c|c|c|c|}
\hline & $c(\mathrm{~cm})$ & $f(H z)$ & $2 h_{\alpha} / c$ & 2.5 \\
$R e_{\text {wing }}$ \\
\hline $\begin{array}{l}\text { Fruit Fly: } \\
\text { Drosophila virilis }\end{array}$ & 0.15 & 240 & 2.8 & 1900 \\
\hline $\begin{array}{l}\text { Honey Bee: } \\
\text { Apis mellifica }\end{array}$ & 0.43 & 240 & 2.8 & 4800 \\
\hline $\begin{array}{l}\text { Bumble Bee: } \\
\text { Bombus terrestris }\end{array}$ & 0.73 & 156 & 3.6 & 6400 \\
\hline $\begin{array}{l}\text { Hummingbird: } \\
\text { Archilochus colubris }\end{array}$ & 1.5 & 52 & 2.6 & 6700 \\
\hline $\begin{array}{l}\text { Hawkmoth: } \\
\text { Manduca Sexta }\end{array}$ & 2.5 & 27.3 & 3.6 & 15000 \\
\hline $\begin{array}{l}\text { Hummingbird: } \\
\text { Patagona gigas }\end{array}$ & 4.3 & 15 & & \\
\hline
\end{tabular}

Table 2. Selected data ${ }^{40,70}$ on time and length scales encountered in nature. The examples listed do not capture the upper or lower bounds of any category listed, but do provide a window in which many of the animals and insects capable of hovering flight are within.

\section{Design of Experiment}

The DOE used a face centered cubic design $(\mathrm{FCCD})^{47}$ and then Latin hypercube sampling (LHS) ${ }^{41}$ to appropriately fill in the remainder of the design space. The reasoning behind this is that a $2^{\text {nd }}$ order polynomial response surface construction has $(\mathrm{N}+1)(\mathrm{N}+2) / 2$ coefficients, $\mathrm{N}$ being the number of variables, and in general, one wants twice this many data points for a proper curve fit. A FCCD design provides $2^{\mathrm{N}}+2 \mathrm{~N}+1$ points: $2^{\mathrm{N}}$ corner points, $2 \mathrm{~N}$ face points, and one center point. Thus for three design variables, FCCD provides 15 of the 20 points required. The LHS then provides a method for efficiently choosing the rest of the points by maximizing the distance between the added points, though by no means is it the only alternative ${ }^{41}$. A tabulation of the $2 \mathrm{D}$ and $3 \mathrm{D}$ simulations run and their respective outcomes are found in the appendix.

\section{Polynomial Response Surface}

The polynomial response surface ${ }^{42}$ is based on regression analysis. The true objective, $y$, is broken up into the predicted response, $\hat{y}$, which takes the form of a polynomial approximation, and the error $\epsilon$ as shown in the equation below. The error is assumed to be independent and though two points may be close, their associated errors need not be.

$$
y(\boldsymbol{x})=\hat{y}(\boldsymbol{x})+\epsilon
$$

For the current study a $2^{\text {nd }}$ order polynomial is used and the predicted response takes the form:

$$
\hat{y}=\beta_{0}+\sum_{i}^{N_{D V}} \beta_{i} x_{i}+\sum_{i}^{N_{D V}} \sum_{j=1, i<j}^{N_{D V}} \beta_{i j} x_{i} x_{j}+\sum_{i}^{N_{D V}} \beta_{i i} x_{i}^{2}
$$

One will have an instance of Eq. (9) for each of the $\mathrm{N}_{\mathrm{TP}}$ training points. The coefficients $\beta$, can be obtained by using the method of least squares. If the system of equations is written in matrix form,

$$
\widehat{\boldsymbol{y}}=\boldsymbol{X} \boldsymbol{\beta}
$$

$$
\boldsymbol{X}=\left[\begin{array}{cccc}
1 & x_{1}^{(1) 1} & \cdots & x_{3}^{(1) 2} \\
\vdots & \vdots & \ddots & \vdots \\
1 & x_{1}^{\left(N_{T P}\right) 1} & \cdots & x_{3}^{\left(N_{T P}\right) 2}
\end{array}\right]
$$

where $x_{C}^{(A) B}$ is the $\mathrm{C}$ design variable, raised to the $\mathrm{B}$ power, at the $\mathrm{A}$ training point, then the matrix $\boldsymbol{\beta}$ can be solved by the following: 


$$
\boldsymbol{\beta}=\left(\boldsymbol{X}^{\prime} \boldsymbol{X}\right)^{-1} \boldsymbol{X}^{\prime} \widehat{\boldsymbol{y}}
$$

\section{Kriging}

The Kriging ${ }^{43}$ methodology predicts the objective function as the sum of a polynomial trend, $f(\mathbf{x})$, and a systematic departure, $\mathrm{Z}(\mathbf{x})$ as expressed below.

$$
\hat{y}(\boldsymbol{x})=f(\boldsymbol{x})+Z(\boldsymbol{x})
$$

The polynomial trend, usually a polynomial of degree 0,1 ,or 2 , accounts for the low frequency general response of the objective function(s) where the systematic departure is relatively localized and catches the higher frequency variation from the trend line but also has a zero mean. Whereas the PRS models assume independent error, Kriging models are built with the assumption the errors are correlated. The Gaussian correlation structure used ${ }^{43}$ takes the form of

$$
\text { Covariance }\left(Z\left(\boldsymbol{x}^{i}\right), Z\left(\boldsymbol{x}^{j}\right)\right)=\sigma^{2} \exp \left(-\sum_{k=1}^{N_{D V}} \theta_{k}\left(x_{i}^{k}-x_{j}^{k}\right)^{2}\right)
$$

One cycles through and evaluates the $\mathrm{k}^{\text {th }}$ design variable $\mathrm{x}$ at the $\mathrm{i}^{\text {th }}$ and $\mathrm{j}^{\text {th }}$ training points. The standard deviation of the design space is denoted by $\sigma$, and $\theta_{\mathrm{k}}$ is a fitting parameter measuring the degree of correlation in the $\mathrm{k}$ direction. Thus, given $\mathrm{N}_{\mathrm{TP}}$ training points, $\mathrm{f}(\mathbf{x}), \sigma$, and $\theta_{k}$, are chosen such that the likelihood function is maximized ${ }^{43}$. As two points move closer, $\left(x_{i}^{k}-x_{j}^{k}\right) \rightarrow 0$, and the correlation function reaches a maximum. Note that at the training points $\hat{y}(\boldsymbol{x})=\mathrm{y}(\mathbf{x})$, and the predicted response matches that of the true response used to train the models.

\section{Radial Basis Neural Network}

Like the Kriging models, the radial basis neural networks use localized correlation functions. However, unlike Kriging, RBNN do not use a global approximation term. The predicted response at the design point $\boldsymbol{x}$ is given below. The weighting factors are denoted $w_{i}$, and response of the $\mathrm{i}^{\text {th }}$ radial basis function is $a_{i}$ which depends on the distance between two design points. The influence of all $\mathrm{N}_{\mathrm{RBF}}$ are summed to get the total response. A popular choice for the basis function is a Gaussian correlation. The parameter $b$ is the bias and inversely related to an input parameter that controls the spread, or radius of influence for the neurons. A spread constant, usually between 0 and 1 , that is higher will cause a smoother transition between neurons' regions of influence and result in a more nonlinear response. Too large a spread constant will diffuse the neurons' sensitivity, and thus a balance must be met.

$$
\begin{gathered}
\hat{y}(\boldsymbol{x})=\sum_{i=1}^{N_{R B F}} w_{i} a_{i}(\boldsymbol{x}) \\
a_{i}=\phi_{R B F}^{(i)}\left(\left\|\boldsymbol{x}-\boldsymbol{x}^{(i)}\right\| b\right) \\
\phi_{R B F}(\eta)=e^{-\eta^{2}}
\end{gathered}
$$

Neurons are added one at a time until a user specified tolerance is achieved. A tolerance that is arbitrarily low is not desirable as this may lead to over-fitting and responses which are not accurate away from the training points. Five percent of the mean data is a common goal tolerance. The number of radial basis functions and their associated weights are solved in a "leave one out" procedure similar to that used in PRESS where parameter combinations are tested until meeting the desired tolerance. Each point is systematically left out and the models constructed, and the fitting parameters resulting in the minimum error as summed from the predicted response to the left out points determine the model used.

\section{Weighted Average Surrogate Models}

Because surrogate models may fit the training data well, does not guarantee a decent fit throughout the design space. Different weighting strategies are employed to minimize the risk of including one of these surrogates, which based on certain error measures may seem acceptable, but in reality gives bad predictions in other parts of the design 
space. The weighted average surrogates currently employed use constant weights, meaning that a certain surrogate will have the same importance throughout the design space. Equation (18) expresses that the (WAS) is a function of the $\mathrm{M}$ candidate surrogates, i.e. those with acceptable PRESS values.

$$
\hat{y}_{W A S}=\sum_{i=1}^{M} w_{i} \hat{y}_{i}
$$

The method proposed by Ref. 60, minimizes the least square error over the domain where $\mathrm{w}$ is the weighting vector, $\mathrm{e}_{\mathrm{WAS}}$ is the error associated with the prediction of the WAS model, and the elements of $\mathbf{C}, \mathrm{c}_{\mathrm{ij}}$ are given as functions of the error of the ith and jth surrogate models. The weights can then be solved for as shown.

$$
\begin{gathered}
M S E_{W A S}=\frac{1}{V} \int_{V} e_{W A S}^{2}(\boldsymbol{x}) d x=w^{T} \boldsymbol{C} w \\
c_{i j}=\frac{1}{V} \int_{v} e_{i}(x) e_{j}(x) d x \\
w=\frac{\boldsymbol{C}^{-1} \mathbf{1}}{\mathbf{1}^{T} \boldsymbol{C}^{-1} \mathbf{1}}
\end{gathered}
$$

The method proposed by Ref. 61, instead use two parameters to control the weighting strategy $\alpha_{w}$ (recommended value of 0.05 ) and $\beta_{w}$ (recommended value of -1 ) used to control the influence of the average, $\mathrm{e}_{\text {avg, }}$ and the individual PRESS errors, $\mathrm{e}_{\mathrm{i}}$, respectively.

$$
\begin{gathered}
w_{i}^{*}=\left(e_{i}+\alpha_{w} e_{\text {avg }}\right)^{\beta_{w}} ; \alpha_{w}<1, \beta_{w}<0 \\
w_{i}=w_{i}^{*} / \sum_{i} w_{i}^{*} \\
e_{\text {avg }}=\sum_{i=1}^{N_{S M}} e_{i} / N_{S M}
\end{gathered}
$$

\section{Global Sensitivity Analysis}

The global sensitivity analysis (GSA) is in general useful for: (i) Determining if a variable is particularly influential in the design space, if not perhaps the variable can be fixed and the degrees of freedom and complexity of the problem reduced. (ii) Ranking the importance of the design variables. (iii) Quantifying the degree of coupling between design variables. For example, is the influence on the design space mostly an individual effort, or is there an effect caused by the interaction of variables?

Sobol's method ${ }^{41}$ is used to for the global sensitivity evaluations. The surrogate model can be written as:

$$
f(x)=f_{0}+\sum_{i} f_{i}\left(x_{i}\right)+\sum_{i<j} f_{i j}\left(x_{i}, x_{j}\right)+\cdots
$$

Once this decomposition has been calculated the total variance,

and partial variances, e.g.,

$$
D=\int f(\boldsymbol{x})^{2} d \boldsymbol{x}-f_{0}^{2}
$$

$$
D_{1}=\int f_{1}(\boldsymbol{x}) d x_{1} d x_{2} d x_{3}
$$

can be calculated. In this fashion, individual contributions, such as $\mathrm{D}_{1} / \mathrm{D}$, or combinations of variables, e.g., $\mathrm{D}_{12} / \mathrm{D}$, can be quantified, effectively capturing the sensitivity of the variable(s) under consideration. 


\section{Results and Discussions}

\section{A. Solution Sensitivities}

Prior to running the DOE simulations, grid sensitivity tests were performed for a representitive case. The 3D flat plate computations used a structured grid next to the flat plate and then an unstructured grid away from the flat plate to reduce the compuational loads, see Figure 2. A representative case, i.e. $R e=100,2 h_{a} / c=3.0, \alpha_{a}=45^{\circ}, \varphi=90^{\circ}$, on grids consisting of approximately $4 \times 10^{5}, 1.4 \times 10^{6}$, and $4.5 \times 10^{6}$ cells were computed to assess the level of sensitivity to the grid. To assess the temporal sensitivity three time steps are used: 500, 1000, and 2000 time steps per period. The spatial and temporal sensitivity results are shown in Figure 3. All of the grids were in agreement, and as such the coarsest 3D flat plate grid was utilized for the surrogate modelling exercise. The temporal sensitivity results confirmed that $\mathrm{T} / \mathrm{dt}=500$ for the time step is sufficient, thus for the $3 \mathrm{D}$ flat plate computations the time step was set to $\mathrm{T} / 500$.

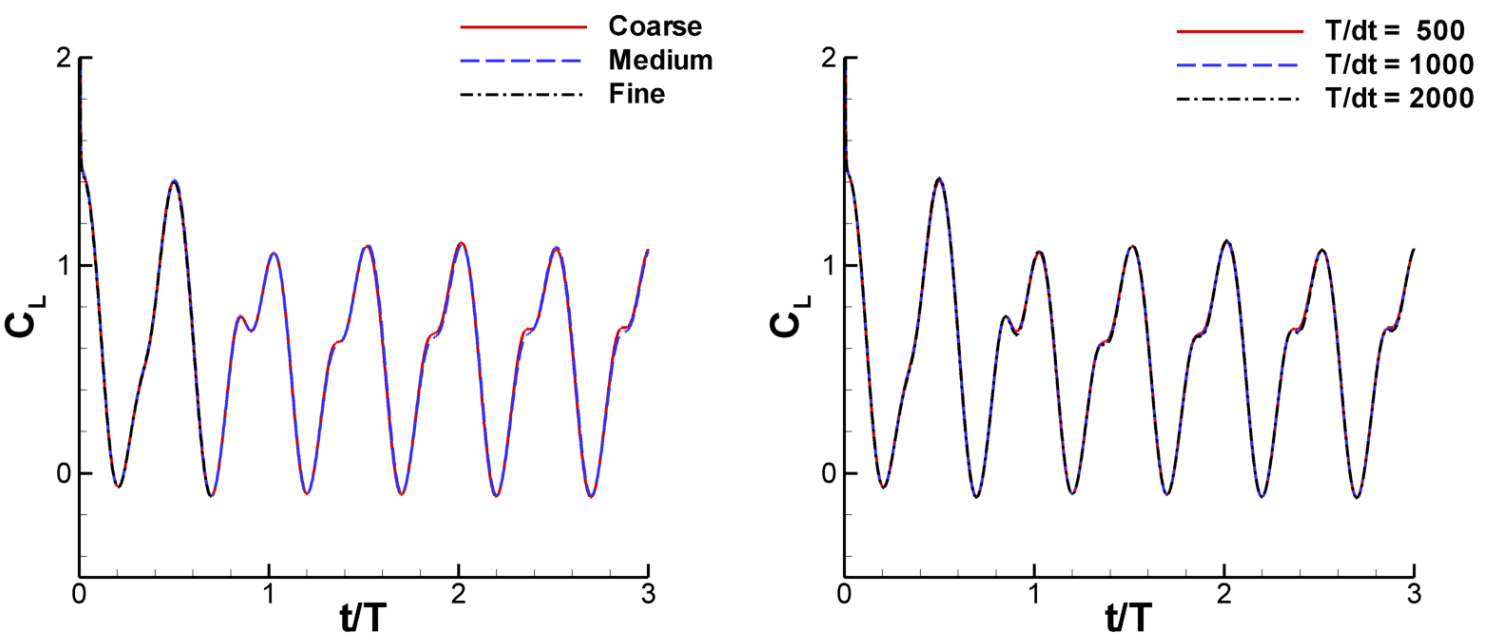

Figure 3. (Left): 3D flat plate grid sensitivity for $\operatorname{Re}=100,2 \mathrm{~h}_{\mathrm{a}} / \mathrm{c}=3.0, \quad \alpha_{\mathrm{a}}=45^{\circ}, \varphi=90^{\circ} . \mathrm{Coarse}^{\circ} \sim 4 \times 10^{5}$ cells, Medium: $\sim 1.4 \times 10^{6}$ cells, Fine: $\sim 4.5 \times 10^{6}$ cells. (Right): 3D flat plate temporal sensitivity for $\operatorname{Re}=100,2 \mathrm{~h}_{\mathrm{a}} / \mathrm{c}=3.0, \alpha_{\mathrm{a}}=$ $45^{\circ}, \varphi=90^{\circ}$ on the coarse grid.

The 3D simulations did not take more than a few cycles to reach an asymptotic behavior and force measurements were taken starting with the $4^{\text {th }}$ cycle.

\section{B. Force Interpretation}

To better understand the implications and limitations of the surrogate modeling results an example is presented of a representative normal hovering case of $2 \mathrm{D}$ ellipse at a $\mathrm{Re}$ of 100 , see Figure 4 . In the flapping wing aerodynamics the unsteady mechanisms such as wake-capturing, delayed stall, and jet interaction are well established in the literature and corresponding 3D flow features will be shown.

The discussion following is generally applicable to the cases where $\phi=90^{\circ}$ with slight modifications. For cases where the phase lag dictates advanced rotation $\left(\phi>90^{\circ}\right)$ or delayed rotation $\left(\phi<90^{\circ}\right)$, the same ideas can be extended though like the parameter suggests, the translation and rotation will be out of phase. Since the kinematics are governed by the sin function, the ends of translation will be found at the non-dimensional times ending at $\mathrm{t} / \mathrm{T}=$ $\mathrm{X} .25$ and $\mathrm{t} / \mathrm{T}=\mathrm{X} .75$. The backstroke starts at X.25 and finishes at X.75. The following forward stroke starts at X.75 and ends at $(\mathrm{X}+1) .25$. The cycle can be broken up into four overlapping regions defined by the unsteady flow mechanisms present.

The first region starts at point 1, once again referring to Figure 4 which is near a local minimum in the lift. As the airfoil is vertical at this point, one would generally expect zero lift. As time continues the airfoil turns back into its previous trajectory which is commonly referred to as wake capturing, points 1, 2, and 3. The peak seen at point 3 will be referred to as the wake capturing peak. Flow field shots of vorticity, Figure 5, demonstrate the nomenclature more clearly. 

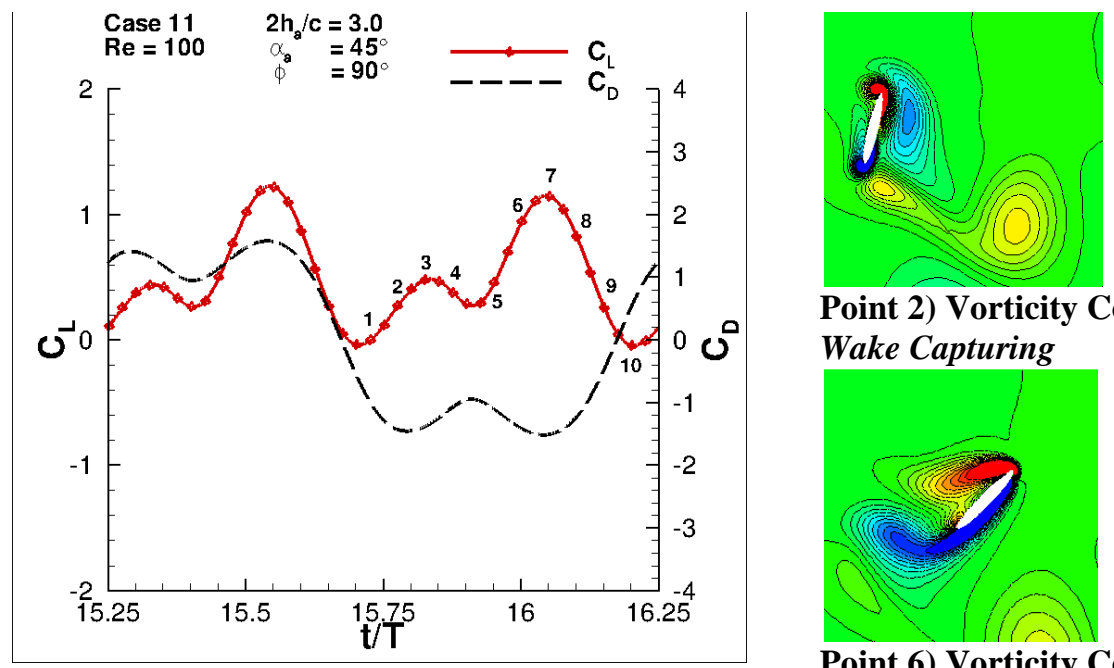

Point 2) Vorticity Contours

Wake Capturing
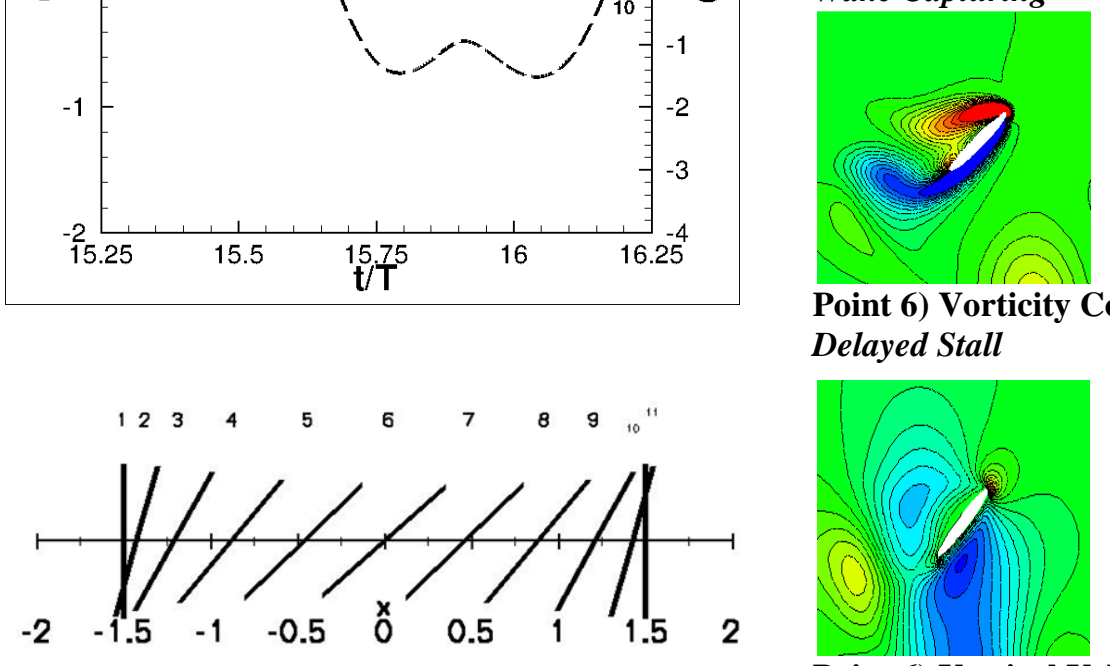

Point 6) Vorticity Contours

Delayed Stall

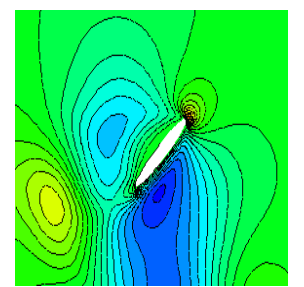

\section{Point 6) Vertical Velocity \\ Jet Interaction}

Figure 4. Illustration of the lift and drag coefficients for a $2 \mathrm{D}$ ellipse normal hovering case with $2 h_{a} / c=3.0, \alpha_{a}=45^{\circ}$, and $\phi=90^{\circ}$ and the corresponding airfoil positions. Three flowfield shots illustrating the unsteady aerodynamics are emphasized.

The second unsteady flow mechanism is due to the angular velocity and the change in circulation about the airfoil, i.e. the Magnus effect. In the literature, one version of this is referred to as rapid pitch-up. In the present study it is seen to both aid and take away from the lift. When $\phi=90^{\circ}$, as in the example, the maximum angular velocities are found at the ends of translation. In 2D this is one of the reasons, the other is a contribution of the jet interaction to be discussed, that explains how negative lift values occur at positive angles of attack. Depending on the kinematic parameters, this unsteady mechanism may interact with any or all of the other unsteady mechanisms.

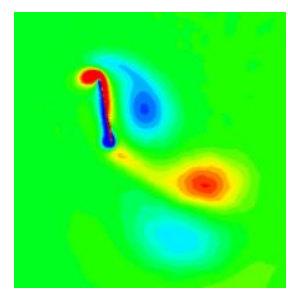

$\mathrm{t} / \mathrm{T}=0.8$

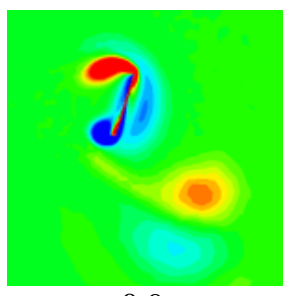

0.9

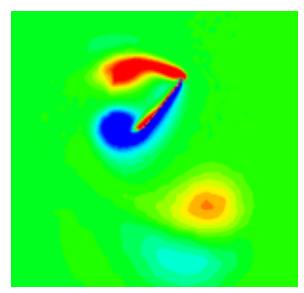

1.0

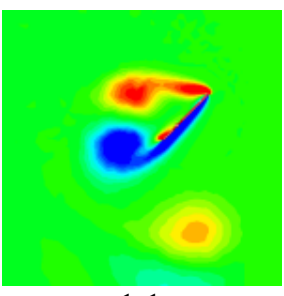

1.1

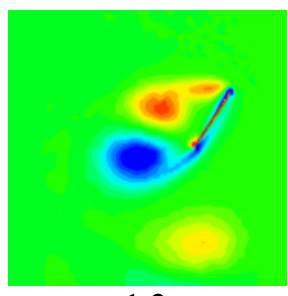

1.2

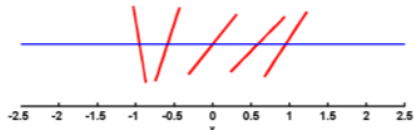

Figure 5. Vortex dynamics in 2D: Vorticity contours (red: counter-clockwise, blue: clockwise) a 2D flat plate at five time instants in one complete forward stroke where $2 h_{a} / c=2.0, \alpha_{a}=45^{\circ}, \phi=60^{\circ}$. The wake-capture mechanism occurs at $t / T=0.8$ and 0.9 . The $L E V$ is clearly visible. 
The third and fourth unsteady flow features overlap significantly. The most commonly known is the delayed stall phenomena here resulting from a leading edge vortex, Figure 5. Specifically, a vortex forms behind the leading edge of the airfoil causing a low pressure region and enhancing lift. Note that in the case illustrated, higher lift is achieved at angles of attack of 45 degrees, an angle well beyond the steady state stall. In cases with higher angular amplitudes, and therefore lower angles of attack, the peak at points 7 and 8 can be reduced significantly because the orientation of the airfoil is not able to promote LEV formation.

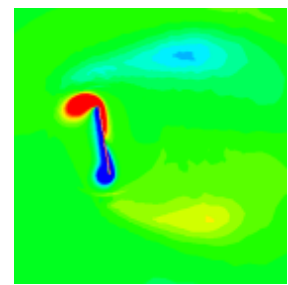

$\mathrm{t} / \mathrm{T}=0.8$

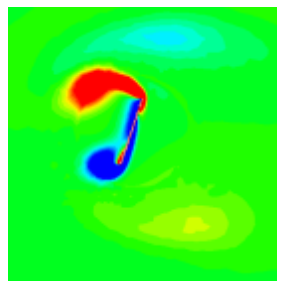

0.9

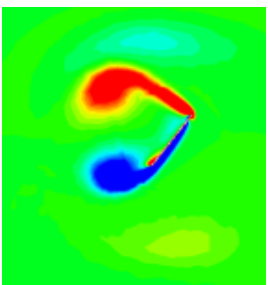

1.0

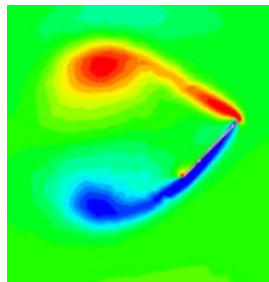

1.1

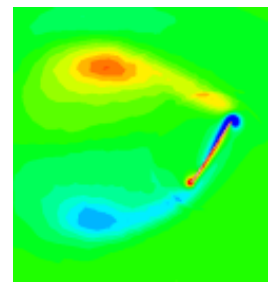

1.2

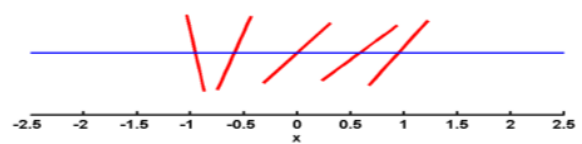

Figure 6. Vortex dynamics in 3D: Vorticity contours (red: counter-clockwise, blue: clockwise) in the symmetry plane of a 3D flat plate $(\mathrm{AR}=4)$ at five time instants in one complete forward stroke where where $2 h_{a} / c=2.0, \alpha_{a}=45^{\circ}$, $\phi=60^{\circ}$. The LEV and the delayed stall mechanism are clearly visible. The wake-capture mechanism is qualitatively different in this case because the shed LEV and TEV convect away from each other.

In 3D flow fields, similar unsteady mechanisms are present. At this aspect ratio and Reynolds number both leading edge vortex and trailing edge vortex are shed, see Figure 6. The LEV creates lower pressure region on the top of the flat plate enhancing the lift generation. On the other hand, the shed vortices may convect away from one another unlike the 2D case (Figure 5). The flat plate returning after the end of a stroke will not encounter the diverging vortices, see $\mathrm{t} / \mathrm{T}=0.8$, and 0.9 in Figure 6 .

The flow mechanism that appeared much stronger in the $2 \mathrm{D}$ computations at $\mathrm{Re}=100$ is a jet-like flow feature present in the path of the airfoil where a persistent downward velocity develops. The jet forms as a result of the flow pushed down by the airfoil and sustained by the shed vortices. These regions within the jet are of comparable magnitude to the maximum translational velocity of the airfoil itself and are influential for a large segment of the cycle, in this case roughly from point 4 to point 9 in Figure 7 . The jet also helps to explain the local minimum, near point 5 which will be referred to as the lift valley. If the angle of attack is low (higher angular amplitudes), or the jet strength is stronger (shorter plunging amplitudes mean the jet decays less between encounters) then this lift valley will become deeper. To support the argument of the jet's influence $C_{L}$ has been plotted during the first two cycles as well as the $15^{\text {th }}$ cycle where the differences between previous cycles has largely stopped at the spatial and temporal resolutions used (see Figure 24). Note that because the simulation starts with a large discontinuity in airfoil velocity, and the plots are shifted such that the force histories start when the airfoil is at the end of its translation, the $1^{\text {st }}$ cycle does not imply no wake. Rather it serves to provide credibility to the claim the jet is at least partly responsible for the aerodynamic performance recorded (i.e. the LEV is not the only factor) as it is clear that as the jet changes strength, the aerodynamic characteristics respond in a noticeable fashion. The weaker jet feature in 3D computations at $\mathrm{Re}=100$ helps us separate the influence of these two unsteady mechanisms during the lift valley. 


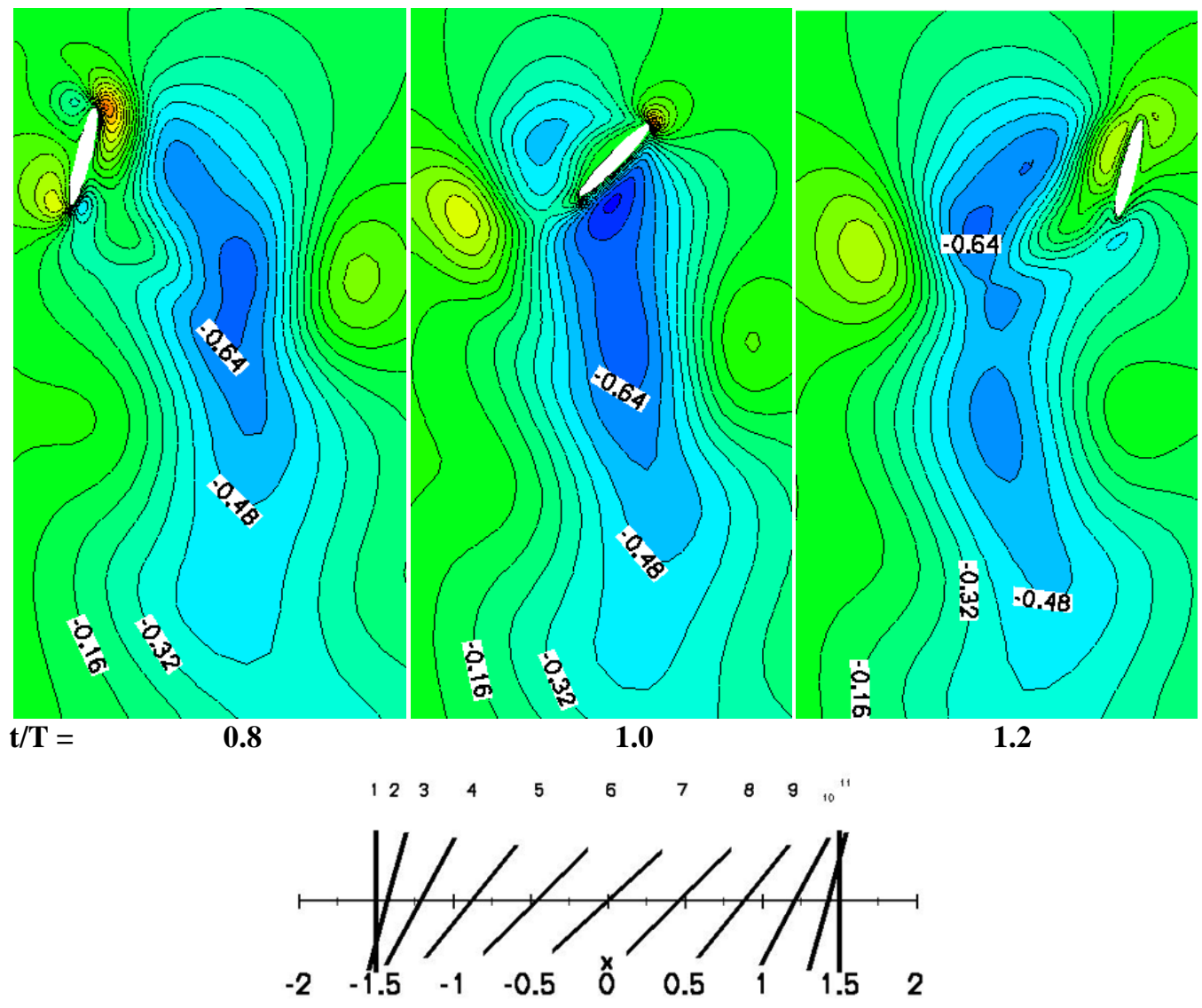

Figure 7. Illustration of the jet flow feature which develops in the path of the airfoil. Velocity contours are displayed (red: positive, blue: negative) with select percentages of the maximum translational velocity marked. These snapshots would correspond to points 2,6 , and 10 respectively after approximately 20 cycles for kinematics of where $2 h_{a} / c=3.0$, $\alpha_{a}=45^{\circ}, \phi=90^{\circ}$.

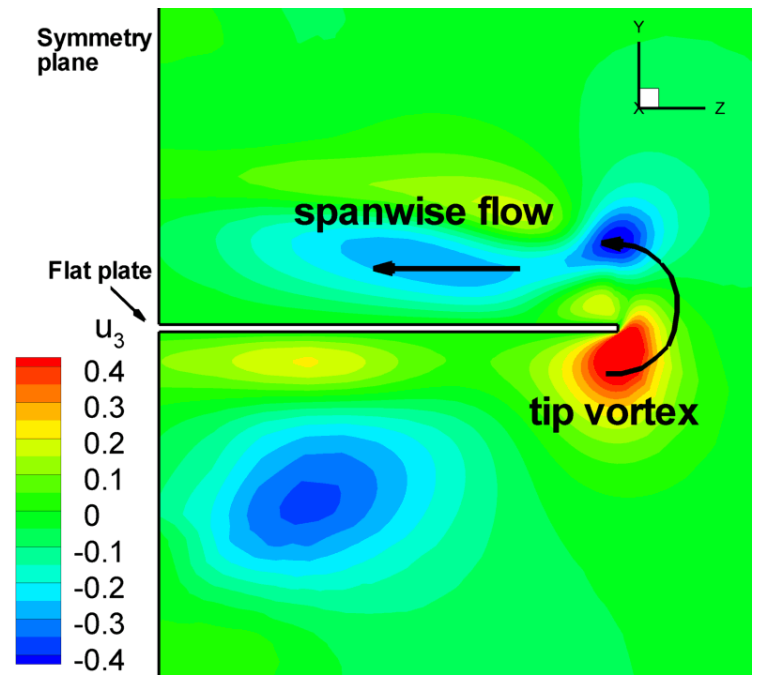

Figure 8. Illustration of the $3 \mathrm{D}$ and finite wing effects of spanwise velocity contours and wing tip vortices. With the symmetry plane on the left and the tip on the right, the 3D flat plat plate features two flow mechanisms not applicable in 2D flow.

In 3D two additional effects, as illustrated in Figure 8, are also encountered. A finite span wing will generate wing tip vortices. These tip vortices will affect the spanwise flow, the $2^{\text {nd }}$ effect, however spanwise flow is seen to 
occur in computations and experiments where tip effects are not present. The spanwise velocity is one factor of understanding the LEV formation as it is sometimes found in the core of the LEV having the effect of stabilizing it.

\section{Wing Shape Sensitivity}

The current study looks at the 3D flow physics about flat plate in an intermediate step towards fluid-structure interactions of a thin wing performing large scale maneuvers. Comparisons are made against 2D simulations of an ellipse stemming from a previous study ${ }^{38}$. Figure 9 shows two cases comparing a 2D flat plate versus a $2 \mathrm{D}$ ellipse, one at high angles of attack and one with aggressive angular velocities. The magnitudes do differ, and the sharp corner of the flat plate does help form a stronger LEV. The lift does vary in amplitude in regions where the LEV dominates, however the trends are the same. The drag is seen to be even less sensitive to the change in shape.
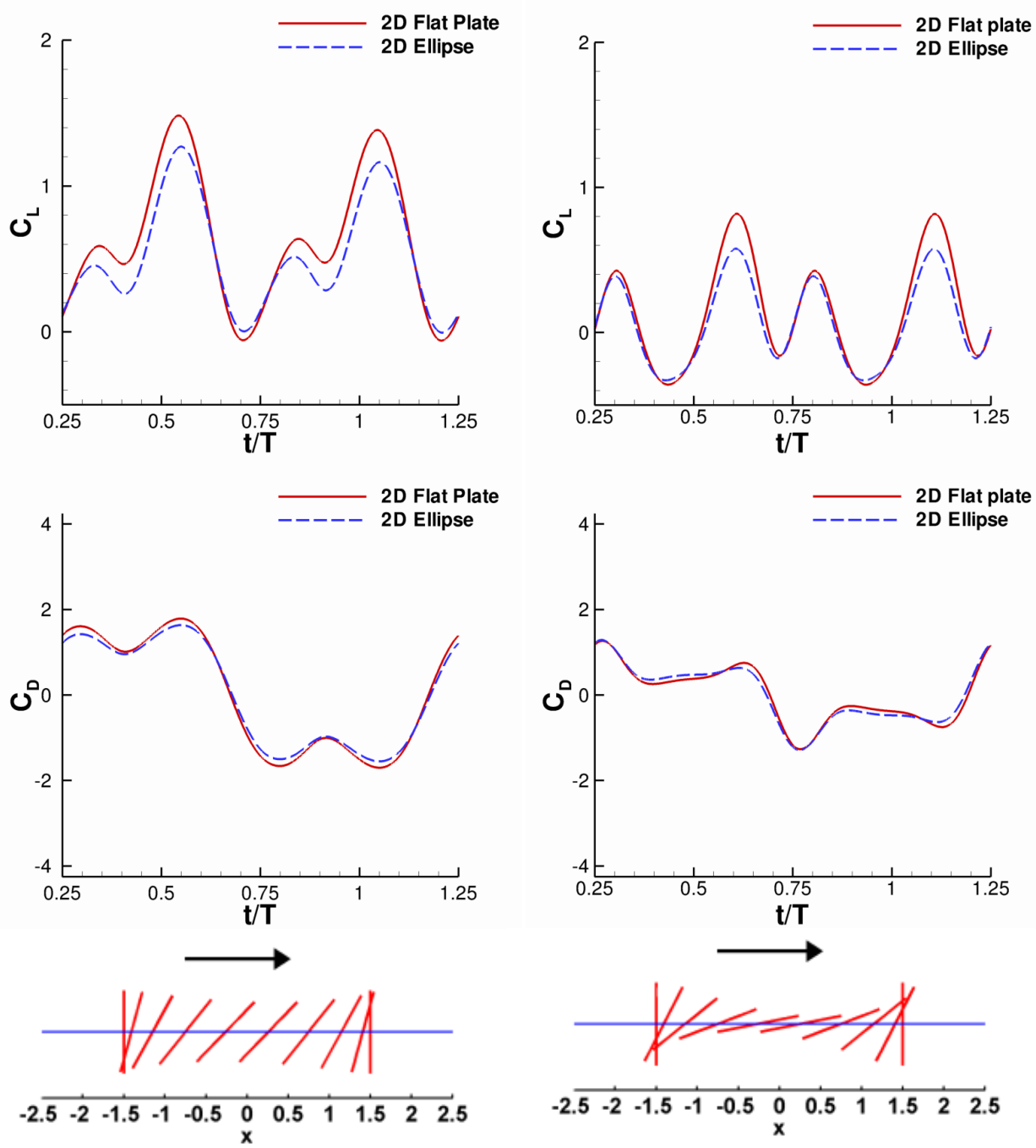

Figure 9. Comparison of forces experienced by a flapping wing at $\mathrm{Re}=100$ of a flat plate and ellipse undergoing identical kinematic motions. Left: $2 h_{a} / c=3.0, \alpha_{a}=45^{\circ}, \phi=90^{\circ}$. Right: $2 h_{a} / c=3.0, \alpha_{a}=85^{\circ}, \phi=90^{\circ}$.

\section{Constructing an Ensemble Surrogate}

When constructing the WAS, the PRESS values can immediately discriminate against bad models. In the tables below the PRESS values for a given surrogate and objective function are presented. The single best model is 
highlighted while the models used in the WAS are bold and italicized. Comments regarding the options used in the surrogate models can be found in the appendix.

The 2D WAS was constructed using Kriging and one of the SVR models. These same two models were also used in 3D, however an additional model, the PRS, was also included because its PRESS evaluations were significantly better than the others when predicting the lift, while comparable to the best models when looking at power.

\begin{tabular}{|l|l|l|l|l|l|l|l|l|l|}
\hline $2 \mathrm{D}$ & KRG & PRS & RBNN & SVR1 & SVR2 & SVR3 & SVR4 & SVR5 & SVR6 \\
\hline $\mathrm{C}_{\mathrm{L}}>$ & $\mathbf{0 . 0 7 5}$ & 0.103 & 0.182 & $\mathbf{0 . 0 4 8}$ & 0.104 & 0.128 & 0.139 & 0.087 & 0.120 \\
\hline $\mathrm{P}_{\text {req }}>$ & $\mathbf{0 . 1 0 7}$ & 0.131 & 0.361 & $\mathbf{0 . 0 6 6}$ & 0.190 & 0.291 & 0.300 & 0.276 & 0.313 \\
\hline
\end{tabular}

Table 3. PRESS values for the 2D computations as predicted by the individual surrogate models for the lift and power. The mean lift was equal to 0.24 and the mean power was equal to 0.56 .

\begin{tabular}{|l|l|l|l|l|l|l|l|l|l|}
\hline 3D & KRG & PRS & RBNN & SVR1 & SVR2 & SVR3 & SVR4 & SVR5 & SVR6 \\
\hline $\mathrm{C}_{\mathrm{L}}>$ & $\mathbf{0 . 0 6 6}$ & $\mathbf{0 . 0 2 8}$ & 0.260 & $\mathbf{0 . 0 6 9}$ & 0.075 & 0.161 & 0.179 & 0.116 & 0.165 \\
\hline $\mathrm{P}_{\text {req }}>$ & $\mathbf{0 . 0 7 3}$ & $\mathbf{0 . 0 7 2}$ & 0.200 & $\mathbf{0 . 0 6 8}$ & 0.196 & 0.293 & 0.302 & 0.295 & 0.322 \\
\hline
\end{tabular}

Table 4. PRESS values for the 3D computations as predicted by the individual surrogate models for the lift and power. The mean lift was equal to 0.36 and the mean power 0.55 .

\section{E. Surrogate Modeling Results}

To start we will look at the general trends seen as the variables changed across the design space. Figure 10 presents the lift coefficient for the $2 \mathrm{D}$ ellipse and $3 \mathrm{D}$ flat plate $(\mathrm{AR}=4)$ simulations at $\mathrm{Re}=100$ as well as the absolute values of their differences. Figure 15 similarly illustrates the power required.

First considering the lift, the general trends found in $2 \mathrm{D}$ are largely still intact in $3 \mathrm{D}$. As the angular amplitude is increased, the average lift decreases. Because higher angular amplitudes correspond to lower angles of attack, this in turn decreases the lift. While not rigorous, two explanations can help justify this. Let us constrict ourselves to 2D and imagine a body whose thickness is small compared to its length, and align this body with the flow. As one raises the angle of attack, the incoming flow gets deflected downwards. This change in momentum is balanced by a lift force on the body. As the angle of attack is increased further, the vertical component of the fluid momentum increases and so will the lift felt by the body. The second component is the effect of the LEV. If the angular orientation and velocity as well as the translational velocity and acceleration are not sufficient enough to form and temporarily sustain a LEV, then the lift that could be extracted from this unsteady mechanism is lost.

The general trend for plunging amplitude also changes little between 2D and 3D. Alter the plunging amplitude and the resulting lift not change much at all. The main difference between 2D and 3D is in magnitude, with the 3D lift being larger than its 2D counterpart's lift over most of the design space.

In contrast, the nature of the surrogate response due to perturbations in phase lag changes significantly. The surrogate response of the differences, see Figure 10 and Figure 11, between the 2D and 3D show the largest gradients in the direction of the phase lag. This would imply that the strength of the 3D effects are sensitive to changes in the phase lag. In $2 \mathrm{D}$ there were regions of the design space where perturbations of phase lag may have had no influence;alternately changes in the phase lag in opposite directions may have had the same end result. The predicted response of lift was a nonlinear function of phase lag. In 3D the response is largely monotonic. We will address these differences shortly.

What can be said quantitatively about the relative impact of the variables? Figure 12 illustrates the total variances due to the respective design variables. Immediately apparent is the change in the hierarchy of design variables. In $2 \mathrm{D}$, the time averaged lift was the most sensitive to the angular amplitude, significantly less sensitive to 
the phase lag, and even less still to the plunging amplitude. While the plunging amplitude was not a negligible influence, an analysis of this sort can not only rank the relative importance but also illuminate variables which do have negligible influence. A finding of this nature can reduce the dimensionality of the design space greatly reducing the time it takes for refinement iterations because that variable can effectively be treated as a constant.

In 3D, the GSA reveals both the plunging amplitude and the phase lag have substantially increased in importance relative to the average lift produced. So much so that the hierarchy of importance changes from angular amplitude being more sensitive than phase lag which is more sensitive than the plunging amplitude in 2D, to 3D where phase lag is the most sensitive, followed by the angular amplitude, and plunging amplitude, while still the least influential of the variables, has a substantially larger influence.

2D

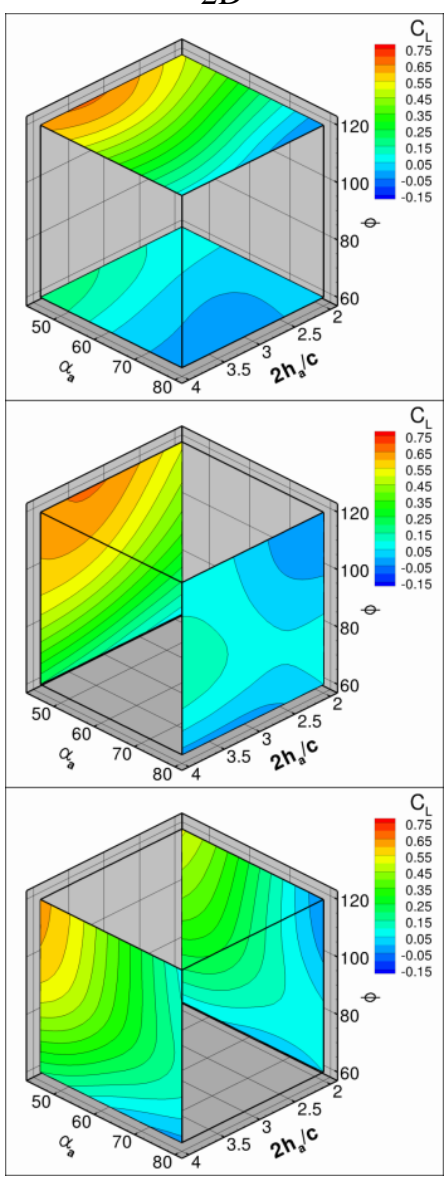

$3 \mathrm{D}$
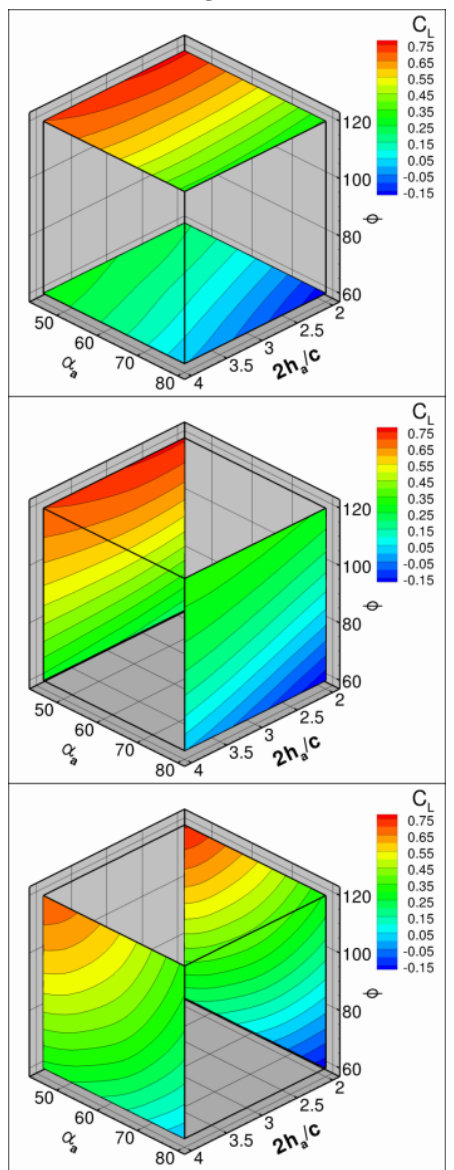

|Difference\|
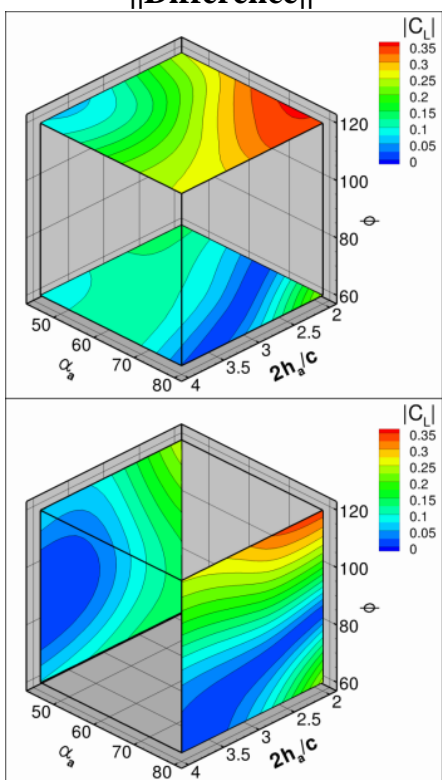

$\alpha_{a}$

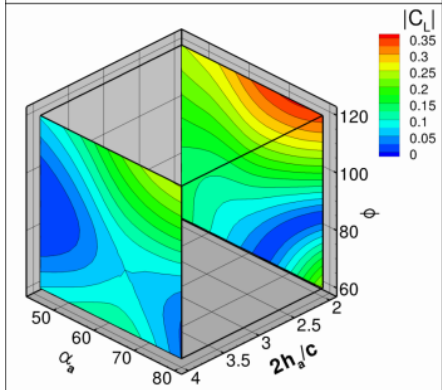

Figure 10. Surrogate modeling results for lift. Left- 2D, Middle-3D, Right- absolute value of the difference between 2D and 3D results. 


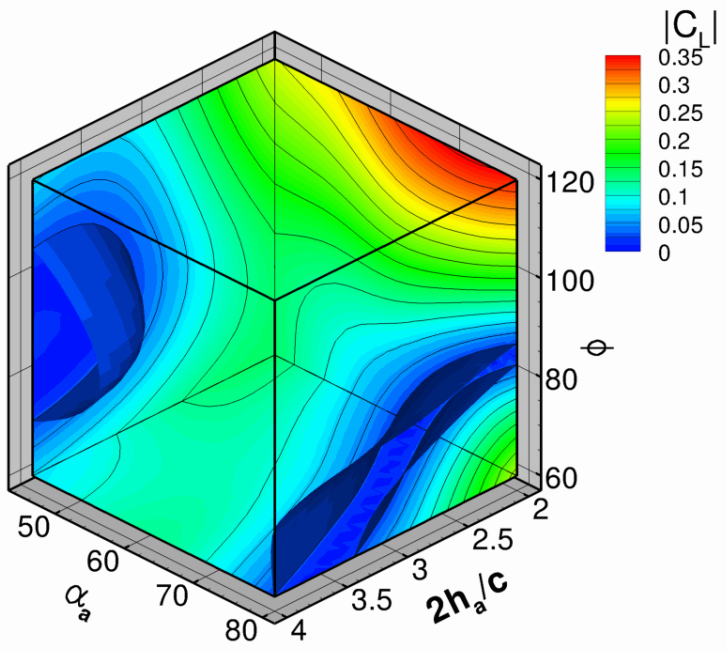

Figure 11. Iso-contours of the surrogate response of the absolute value of the difference between 2D and 3D lift.

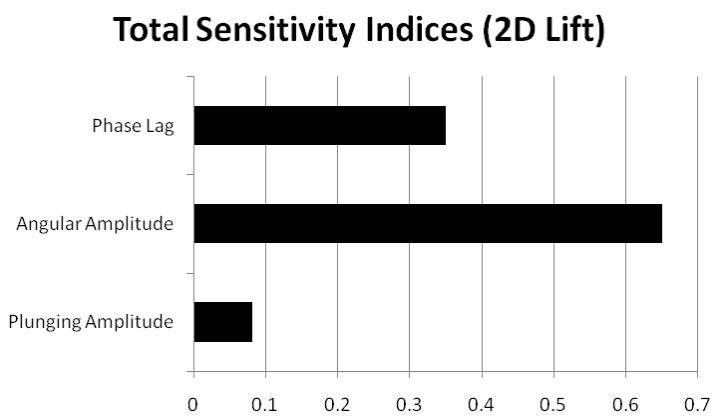

Total Sensitivity Indices (2D Power)

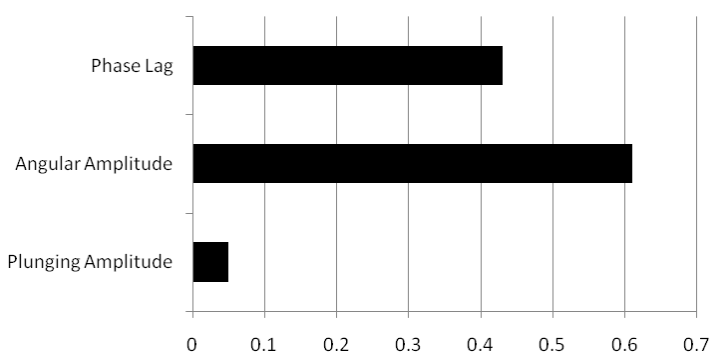

Total Sensitivity Indices ( 3DLift)

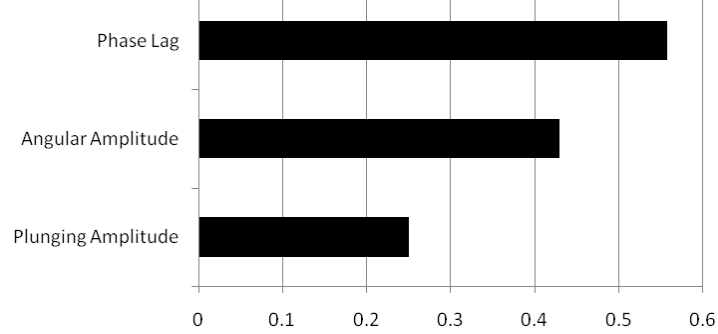

Total Sensitivity Indices (3D Power)

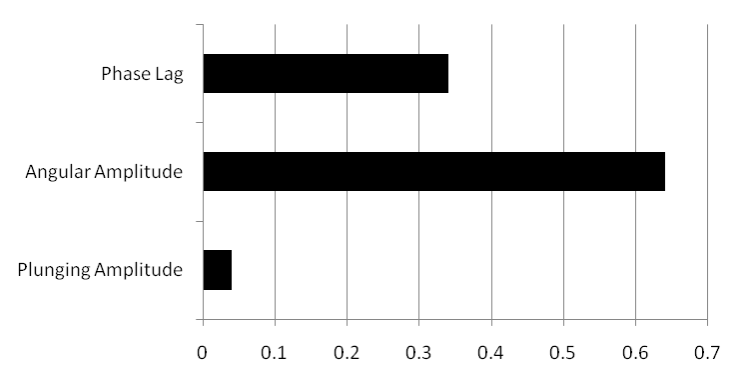

Figure 12. Global sensitivity analysis (GSA) of lift and power for 2D and 3D hovering kinematics.

\section{F. Instantaneous Effects on the Force Coefficients}

To understand where the differences between the 2D behavior and 3D behavior arise, it will prove useful to refer to the time histories of the lift and drag coefficients in Figure 13. The observation that the lift values are generally higher in 3D is seen to take be a consequence of the behavior in the lift valley region between the wake capture and delayed stall peaks. The valley was seen in 2D to be caused by a combination of the jet interaction, which accelerated the flow on the underside of the airfoil, and the downward pitching motion. 

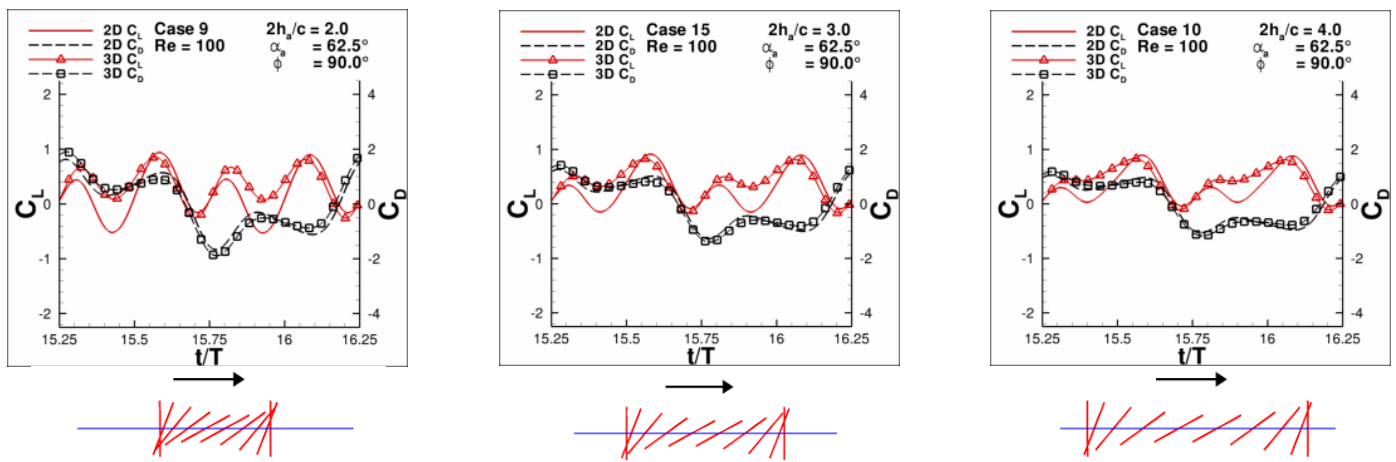

(a)
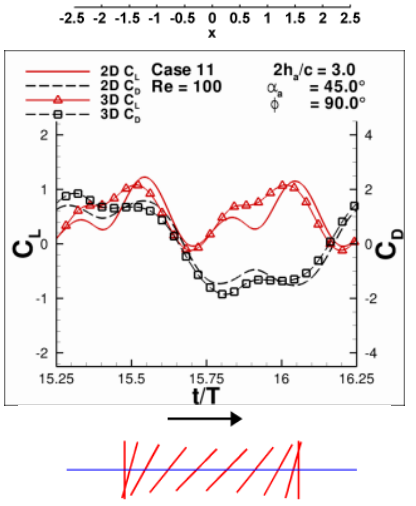

$\begin{array}{lllllllllll}-2.5 & -2 & -1.5 & -1 & -0.5 & 0 & 0.5 & 1 & 1.5 & 2 & 2.5\end{array}$
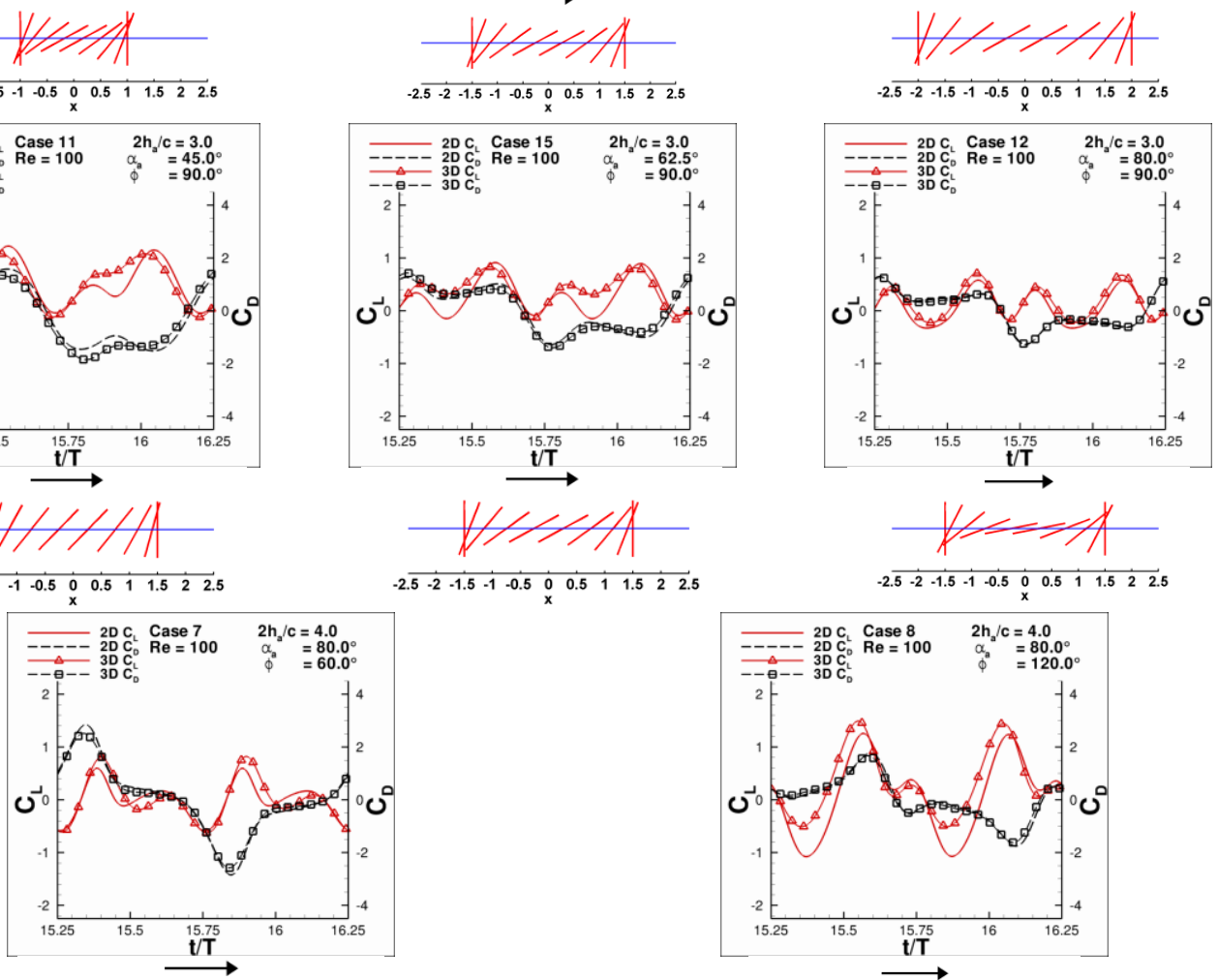

(b)

(c)
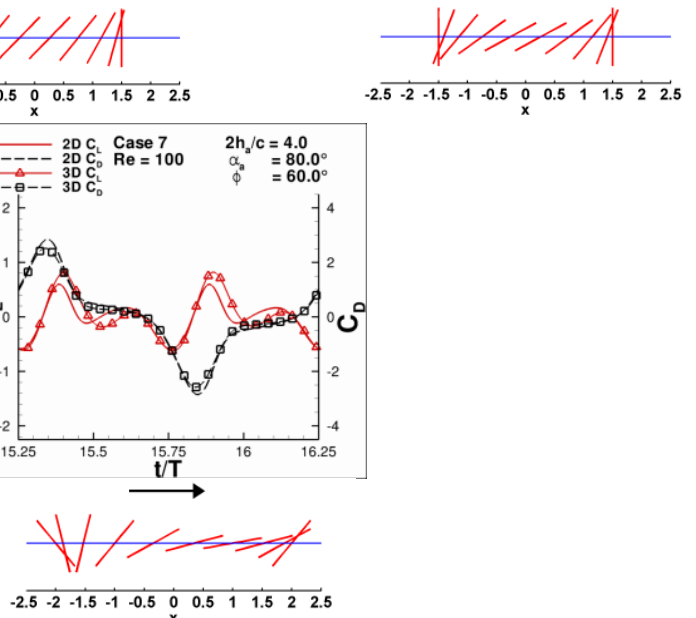

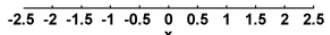
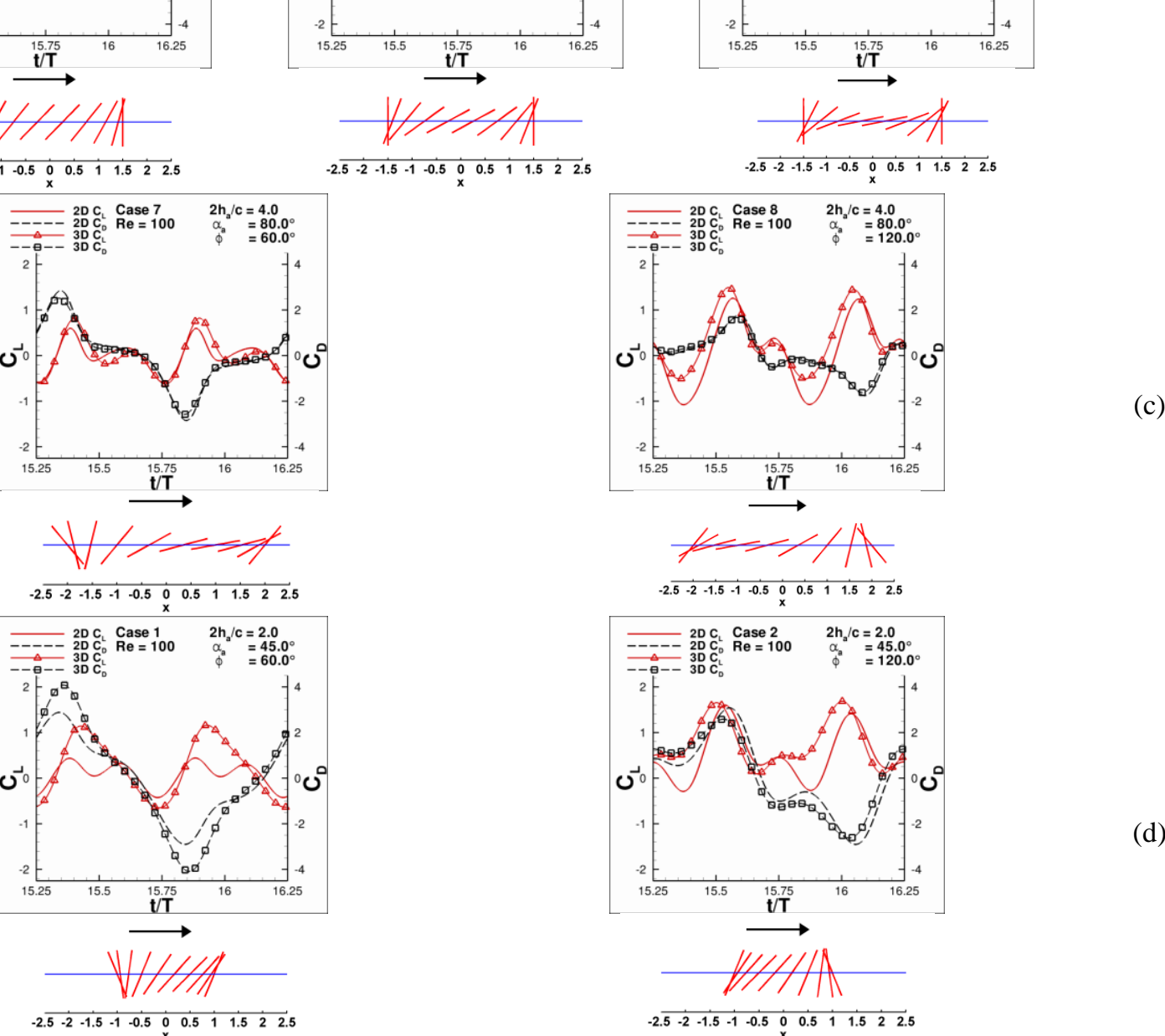

(d)

Figure 13. Time histories of the lift and drag coefficients for selected cases (a) as $2 h_{a} / c$ is increases from 2.0 (left) to 4.0 (right) holding $\alpha_{a}=62.5^{\circ}$ and $\phi=90^{\circ}$ (b) as $\alpha_{a}$ is increased from $45^{\circ}$ (left) to $80^{\circ}$ (right) while holding $2 h_{a} / c=3.0$ and $\phi=90^{\circ}$ (c) examining delayed rotation $\phi=60^{\circ}$ (left) and andvanced rotation $\phi=120^{\circ}$ (right) while holding $2 h_{a} / c=4.0 \alpha_{a}=$ $80^{\circ}$ (d) examining delayed rotation $\phi=60^{\circ}$ (left) and andvanced rotation $\phi=120^{\circ}$ (right) while holding $2 h_{a} / c=2.0 \alpha_{a}=45^{\circ}$. 
In $3 \mathrm{D}$, at $\mathrm{Re}=100$, the jet like flow feature exists near the symmetry plane but does not persist in the wake near the wingtips as the influence of the shed vortices decay too fast to sustain the downward momentum imparted to the flow by the airfoil. This is illustrated in the discussion of the flow physics in Figure 5and Figure 6, most prominently in the vortices shed from a previous cycle for which the 3D vortices have all but disappeared. However the total difference in the amplitude cannot be attributed to only the jet interaction just yet. Three dimensional effects such as the spanwise flow at the core of the LEV have been shown to have a stabilizing effect ${ }^{24}$ which may also have an influence as might the tip effects that will be illustrated later. The rightmost case in Figure 13 (b) would further substantiate the claim that the jet interaction is at least partially responsible for the discrepancy between 2D and 3D lift values near the lift valley. This was one of the few 2D cases which did not produce a persistant downward jet. Note that at a positive angle of attack of $10^{\circ}$ the airfoil is generating negative lift. However, the 2D and 3D cases at $\alpha_{\mathrm{a}}=80^{\circ}$ also could not support the formation of a LEV (see Figure 20), and so the effects cannot be completely separated.

Now we will touch on the discrepancy seen in the lift response due to phase lag. Figure 13 (c) and (d) show the consequence of changing the phase lag in two different regions of the design space. In 2D it was seen that instantaneous lift values could be increased significantly by employing advanced rotation. This was a result of higher angles of attack and positive angular velocities when the airfoil was near its maximum speed. Delayed rotation, while sharing the higher angle of attack at the maximum translational velocity, differed as it had negative angular velocities at that point and subsequently suffered in lift. The advanced rotation, while reaching the highest values of instantaneous lift, also suffered from a deeper lift valley which served to cancel out some of the benefit seen in the delayed stall peak. So while instantaneously the phase lag was visibly an influential factor, the choice of objective function as the time integrated values over the course of a cycle, hid the real influence of the phase lag. Two major resulting effects, one increasing lift, one decreasing lift resulted in a net effect that did not change much in some regions of the design space. In 3D once again the lift valley is not as pronounced and the delayed stall benefit is still intact, and thus we see the source of the monotonic behavior in lift due to phase lag in 3D.

\section{G. Power Required}

Figure 15 displays the 2D ellipse and 3D flat plate surrogate responses of the power required as well as the absolute value of their differences. Figure 14 also highlights the differences. Not only are all the trends quite similar but the magnitudes also compare quite well. This would appear to imply that 3D flow features, those not feasible in 2D, for which lift was sensitive to, do not play a significant role in these kinematic simulations. From the instantaneous force histories in Figure 13, the agreement in drag coefficients is very close except at a combination of low plunging amplitudes with low angular amplitudes and low phase lags. This case exhibits delayed rotation (left example in Figure 13 (d)) and will be looked at in more detail later. The physical reasoning behind the observed trends in 2D and 3D stays the same. During normal hovering, $\phi=90$, the airfoil/wing is perpendicular to the direction of motion at the ends of translation but has little translational velocity. As the phase lag is perturbed in either direction the airfoil can have an appreciable velocity while perpendicular to the direction of motion and whether the body is accelerating when perpendicular (as in delayed rotation cases Figure 13 (c) and (d) on the left) or decelerating (as in advanced rotation, cases Figure 13 (c) and (d) on the right) has a sizable impact on the instantaneous drag that is felt, and consequently the power required for the maneuver.

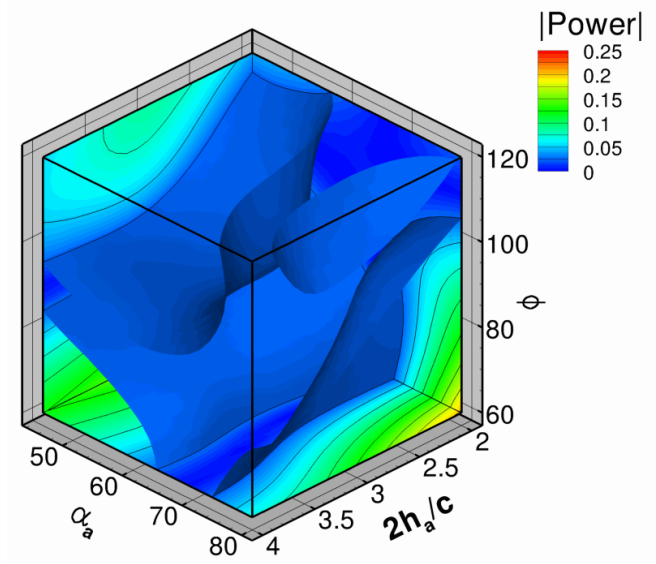

Figure 14. Iso-contours of the surrogate response of the absolute value of the difference between 2D and 3D power required. 
$2 \mathrm{D}$

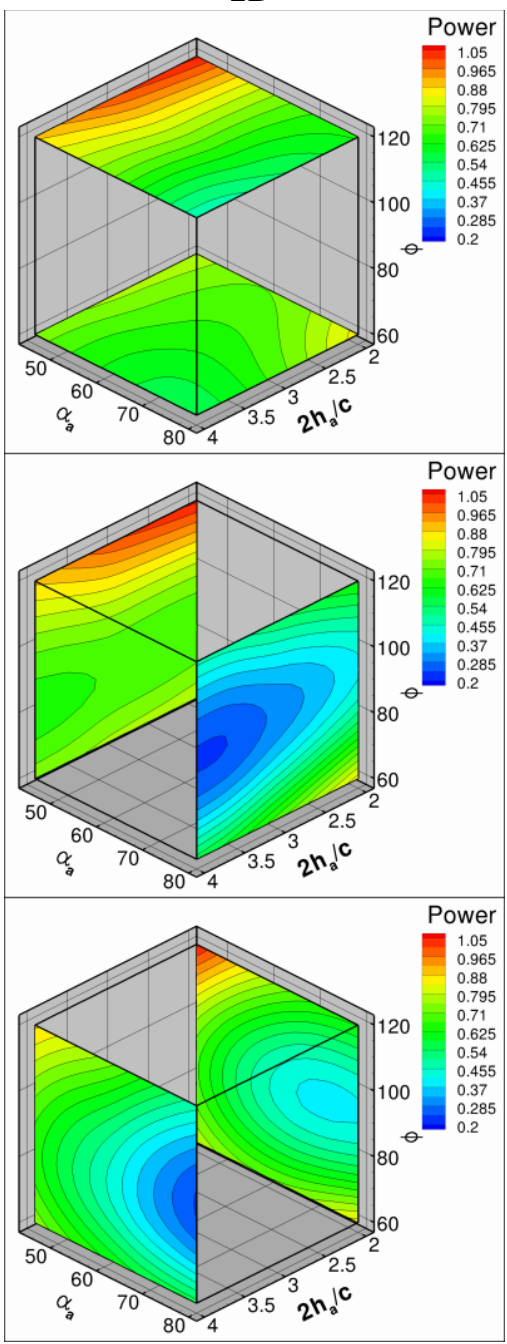

$3 \mathrm{D}$
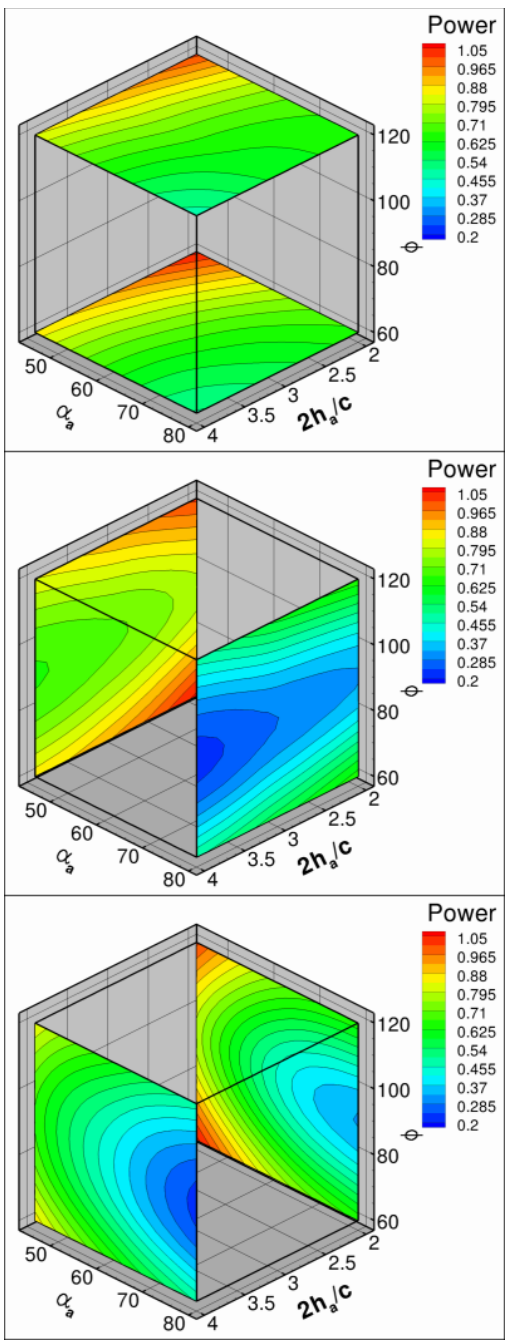

||Difference\|
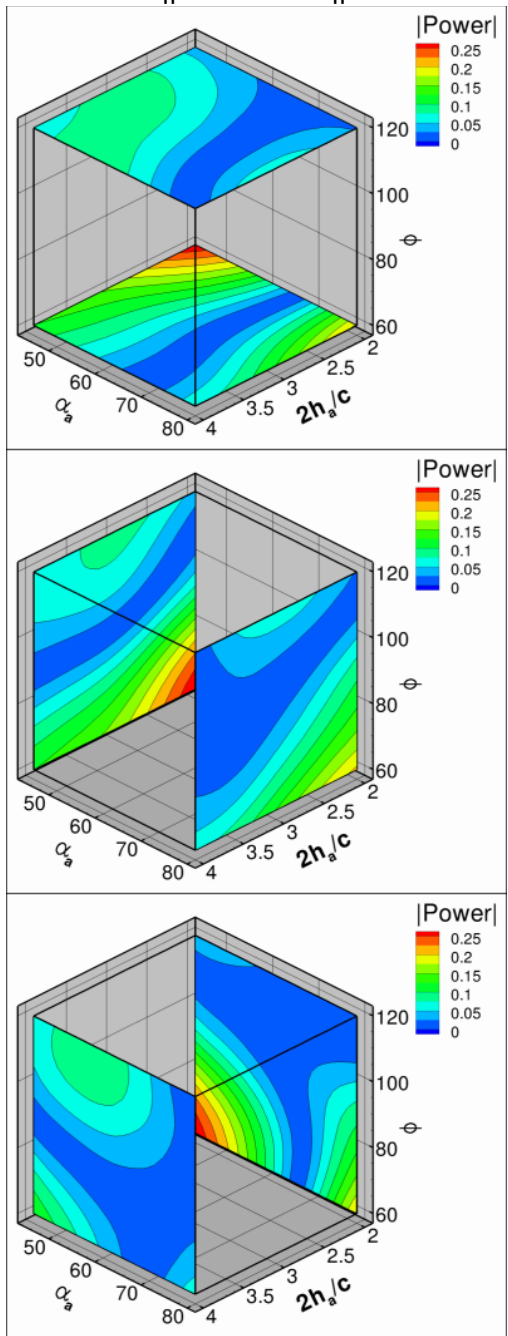

Figure 15. Surrogate modeling results for power required. Left- 2D, Middle-3D, Right- absolute value of the difference between $2 \mathrm{D}$ and $3 \mathrm{D}$ results.

\section{H. Pareto Front}

In engineering design it is often the case that different objectives are in competition. One tool used to evaluate the trade-offs between objective functions is called the Pareto front. The Pareto front consists of non-dominated points and can be thought of as the set of best possibilities, see Figure 16. Non-dominated points can be thought of as points for which one could not improve all objective functions simultaneously. The current objectives are to maximize lift and minimize drag. Points on the Pareto front therefore involve those for which increases in lift are accompanied by increases in drag. Likewise, moving along the curve one finds that decreases in drag are paid for my decreases in lift. It is customary to pose all objectives as minimization expressions. Therefore when the results are plotted, any objective which is maximized, like lift, is multiplied by a negative sign. While not universal, this approach provides consistency in the general look of a "good" solution, e.g. a front which approaches the axes as seen below. Note that the order of the Pareto front increases with the number of objective functions, three objectives would define a three dimensional surface and likewise more objectives would increase the dimension of the Pareto front.

Visualizations are convenient in that they can give a physical sense off the trade-offs involved. The slopes of the lines give an indication of how much one will have to give up in one objective to gain in another. The most striking feature between these two is the fact that the entire 3D Pareto front, and even many points not on the front, 
outperform the 2D Pareto front. What this means is that for at a given lift, there are multiple kinematic combinations in 3D which will yield similar values but with lower power requirements. Conversely for a given power threshold, multiple 3D solutions will yield better lift results than what their 2D counterparts can provide.

The paths through the design space are plotted below their respective Pareto fronts in Figure 16. It is seen that the high lift region follows the lower bound of the angular amplitude suggesting that future iterations should decrease the lower bound for higher lift solutions. Over all the design variable combinations on the optimal front are comparable.

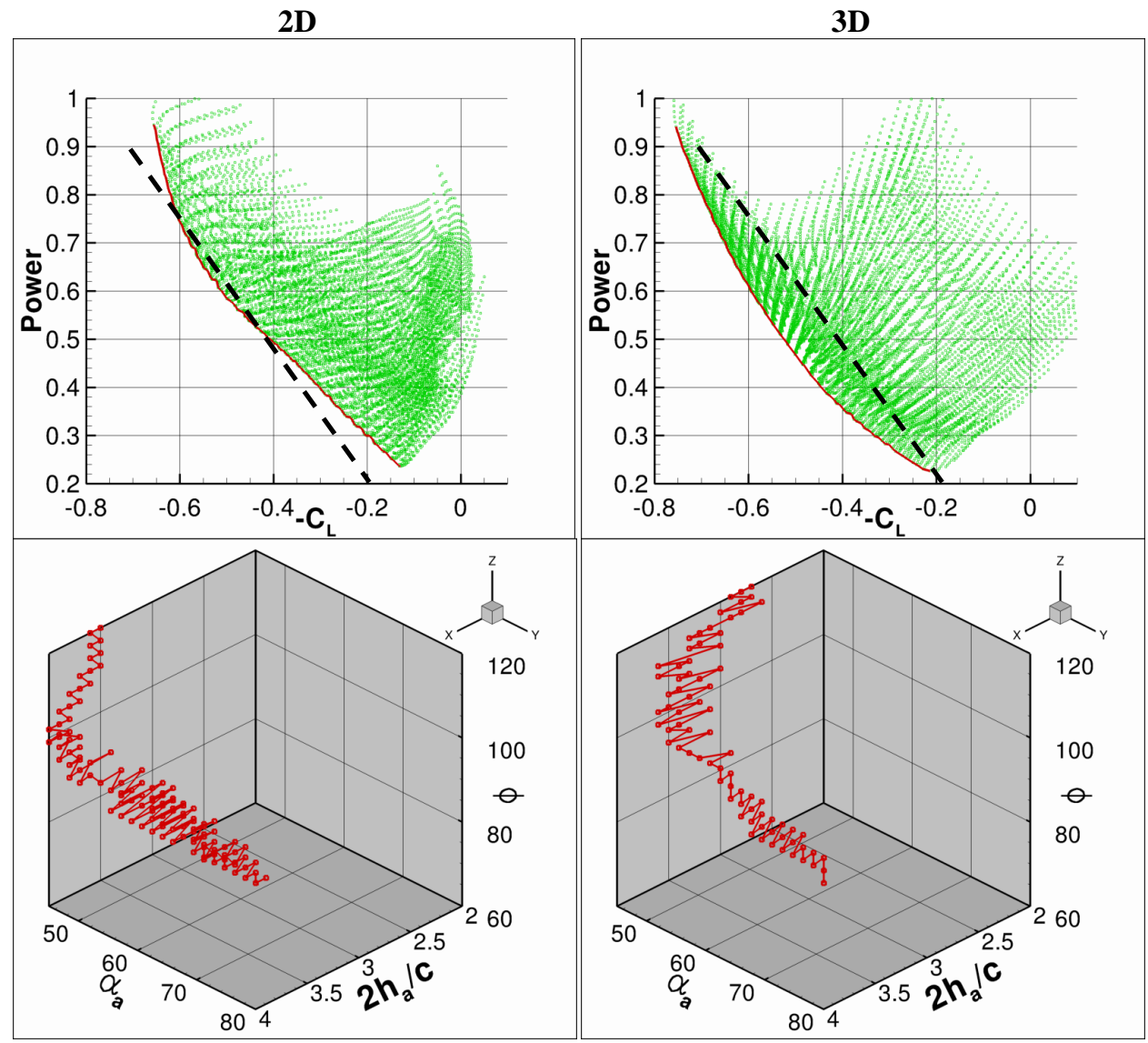

Figure 16. Pareto fronts illustrating the competing objectives of lift and power requirements in 2D (left) and 3D (right) and the design variable combinations which provide those fronts.

\section{Highlights of 3D Flow Physics}

The 3D flow field for $\mathrm{Re}=100$ for the flat plate with aspect ratio of 4 is highlighted by considering two cases, case 1 , and case 12, see the Appendix. Case 1 is characterized by the relatively large difference between the 2D flat plate and 3D flat plate time history of lift coefficient, and the case 12 by relatively similar 2D flat plate and 3D flat plate time history of lift coefficient, see Figure 17. We focus on the four unsteady mechanisms that we identified in 2D hovering: delayed stall, jet interaction, rapid pitching motions, and wake-capture.

\section{Case $1-$ Different $2 D$ and $3 D$ time history of $C_{L}$}

Figure 18(b) shows the contours of the z-vorticity in the symmetry plane of the flat plate at five time instants in forward stroke for case 1 . The decreasing angle of attack, and increasing translational velocity from $\mathrm{t} / \mathrm{T}=0.8$ to 1.0 contribute to the vorticity generation. Compared to the z-vorticity contours from the $2 \mathrm{D}$ flat plate computation, as shown in Figure 18(a), two qualitative different features are visible. Firstly, the leading edge vortex at $t / T=0.9$, and 1.0 are larger in 3D flat plate flow. Secondly, the leading edge, and the trailing edge vortices are shed along diverging angles in the 3D flat plate case, while in the 2D flat plate result the two vortices convect to each other. The latter behavior leads to different wake-capture mechanisms in the 2D and 3D flow. In the 2D flat plate case the shed vortices stay en route of the flat plate, such that the flat plate moves into the wakes created in the previous stroke as shown in Figure 18(a) $(\mathrm{t} / \mathrm{T}=0.8,0.9)$. On the other hand, in the 3D flat plate case because of the diverging vortices 
the wake-capturing mechanism is qualitatively different. Figure $18(\mathrm{~d}, \mathrm{e})$ show the vertical velocity contours for the 2D flat plate, and 3D flat plate computations in the symmetry plane, respectively. In the 3D result the leading edge vortex shed in the previous stroke, and the leading-edge vortex being developed interact with each other at $\mathrm{t} / \mathrm{T}=0.8$, and 0.9 to create larger upward jet, than in the 2D flat plate case where the position of the wake shed in the previous stroke is different.
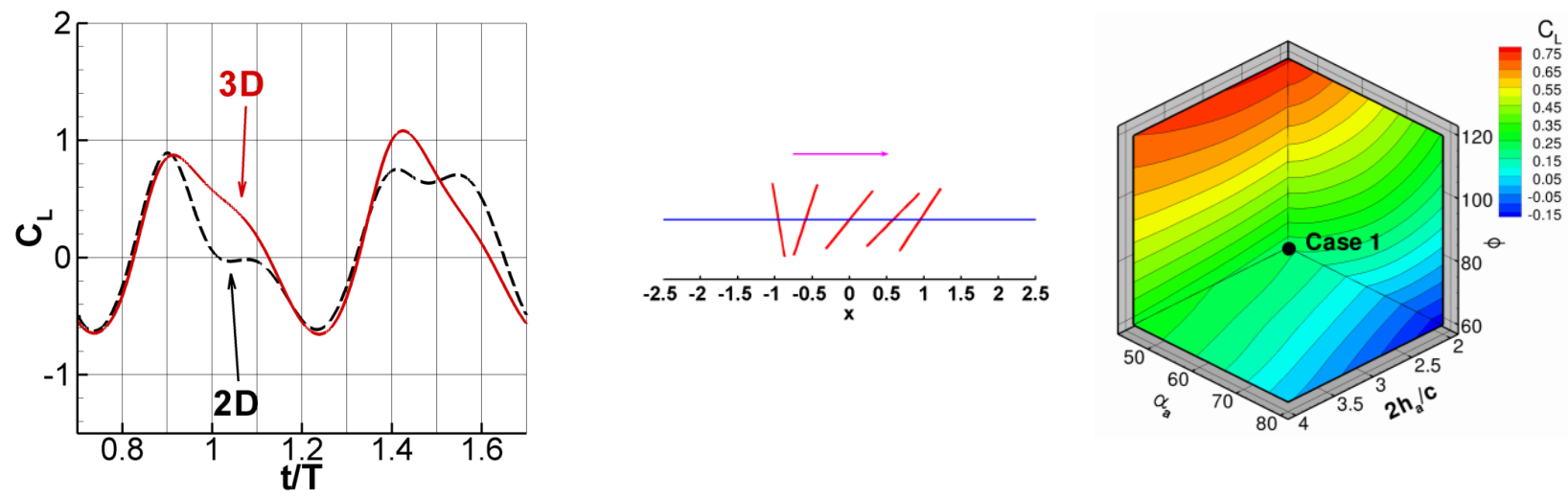

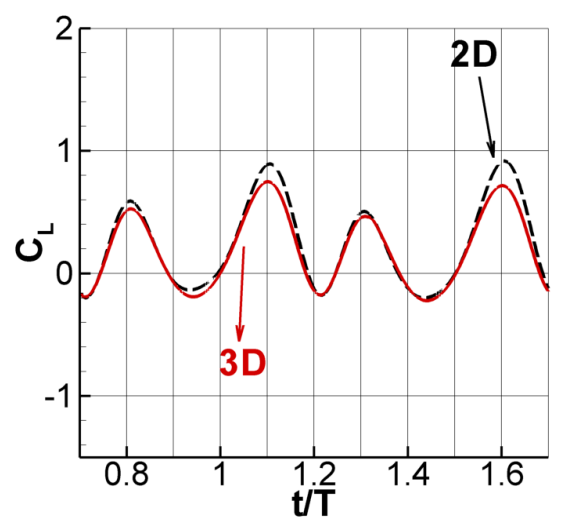

(a) Time history of lift coefficient in $2 \mathrm{D}$ and $3 \mathrm{D}$

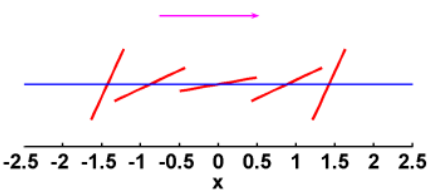

(b) Schematics of applied kinematics

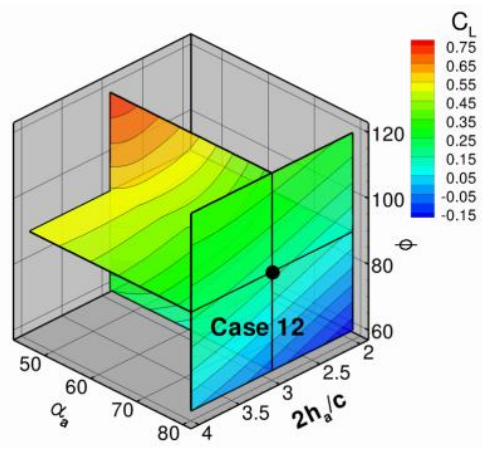

(c) Locations in the design space

Figure 17. Time histories, schematics of kinematics, and the locations of the kinematics in the surrogate design space at t/T $=0.8,0.9,1.0,1.1$, and 1.2 for the case $1\left(h_{a}=2.0, \alpha_{a}=45^{\circ}, \phi=60^{\circ}\right)$, and the case $12\left(h_{a}=3.0, \alpha_{a}=80^{\circ}, \phi=\right.$ $\left.90^{\circ}\right)$.

Figure 18(c, f) show the z-vorticity, and the vertical velocity contours, respectively, near the wingtip at $\mathrm{z} / \mathrm{c}=1.8$. For these kinematics the spanwise variation is substantial as we compare to the flow in the symmetry plane to the flow in at $\mathrm{z} / \mathrm{c}=1.8$. Similar behavior in spanwise variation can be seen in Figure 19 where iso-Q surfaces are used to visualize the vortex structure, and the spanwise lift distribution from pressure per unit length ${ }^{4}$ at $t / T=0.8,0.9,1.0$, 1.1 , and 1.2 are shown. The generation of the tip vortex creates additional negative pressure region on the top surface of the flat plate enhancing the spanwise lift near the wingtips. At $t / T=0.8$, and 0.9 the spanwise lift due to tip vortex is still smaller than in the symmetry plane, and the spanwise lift distribution plots in Figure 19(b) show convex shape. On the other hand at $\mathrm{t} / \mathrm{T}=1.0,1.1$, and 1.2 the spanwise lift in the symmetry plane is larger than near the wingtip causing concave shape for the distribution.

An interesting phenomena seen is the repelling of the LEV and TEV. The tip vortex is able to anchor the LEV at the tips, however the LEV away from the tip region is not strong enough to latch on as the airfoil translates away. The tip vortices feed flow onto the top side of wing which then serves to further separate the vortices which in turn changes the influence vortices, see Figure 18 (b) and (c).

\footnotetext{
${ }^{4}$ In the following discussion the spanwise lift distribution will refer to the spanwise lift distribution from pressure.
} 
Vertical Velocity: $\quad-0.8-0.6-0.4 \quad-0.2 \quad 0 \quad 0.2 \quad 0.4 \quad 0.6 \quad 0.8$
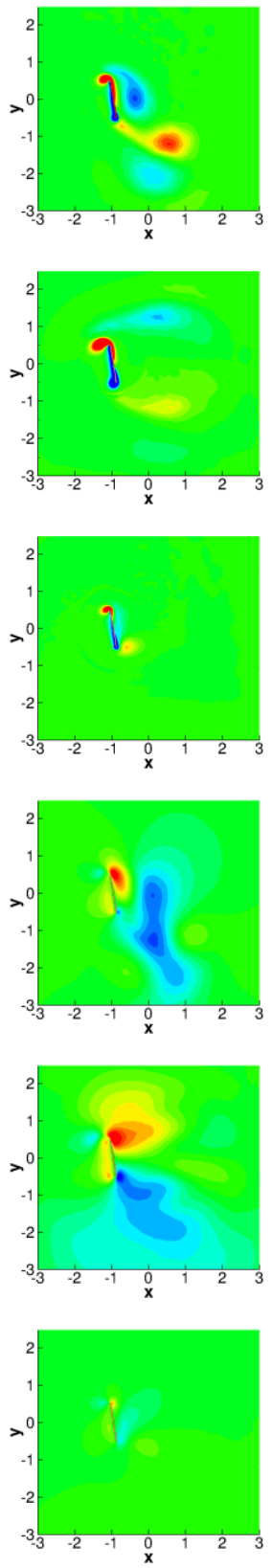

0.8
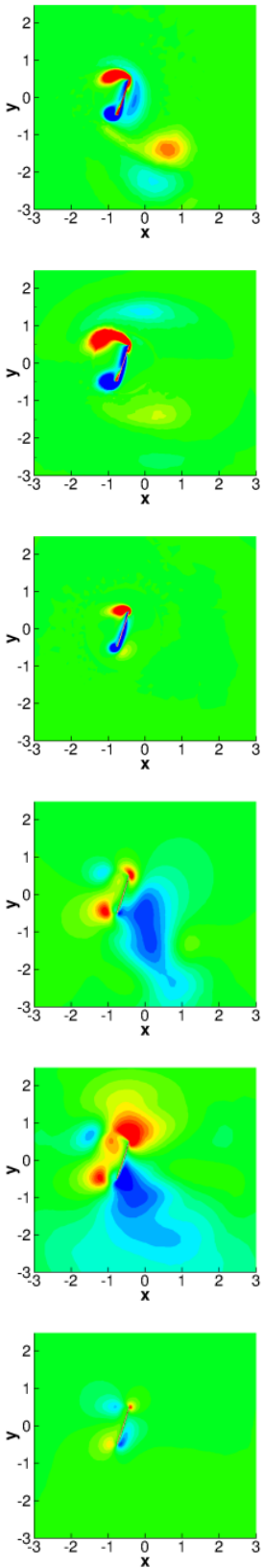

0.9
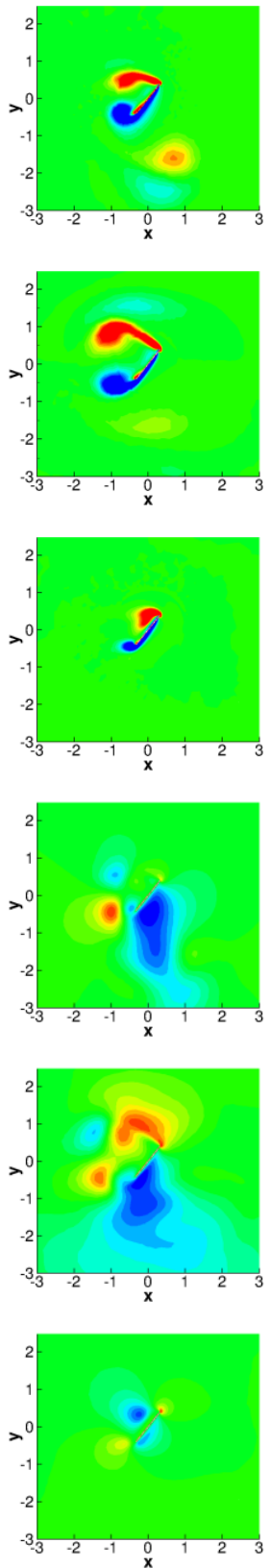

1.0

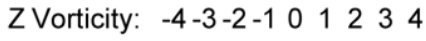
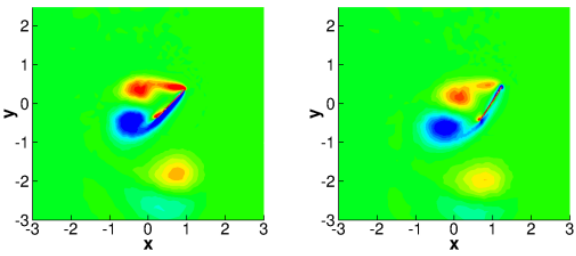

(a)
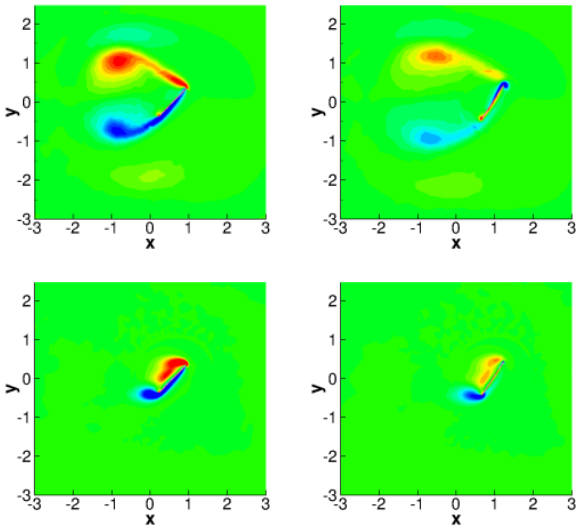

(c)
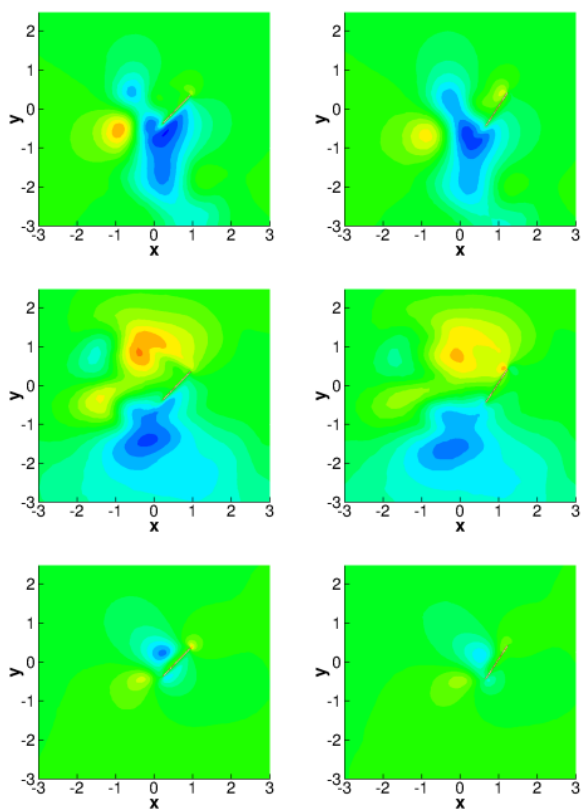

1.1

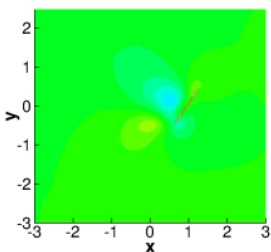

1.2 (b)

(d)

(e)

(f)

T

Figure 18. Contour plots at five time instants in the forward stroke, i.e. $t / T=0.8,0.9,1.0,1.1$, and 1.2 , for the case 1 $\left(h_{a}=2.0, \alpha_{a}=45^{\circ}, \phi=60^{\circ}\right)$ from 3D and 2D computations: (a) z-vorticity contours from 2D computation; (b) zvorticity contours in the symmetry plane of 3D computation; (c) z-vorticity contours near the wingtip $(\mathrm{z} / \mathrm{c}=1.8)$ plane from the 3D computation; (d) vertical velocity contours from 2D computation; (e) vertical velocity contours in the symmetry plane of 3D computation; (f) vertical velocity contours near the wingtip $(\mathrm{z} / \mathrm{c}=1.8)$ plane from the 3D computation. 

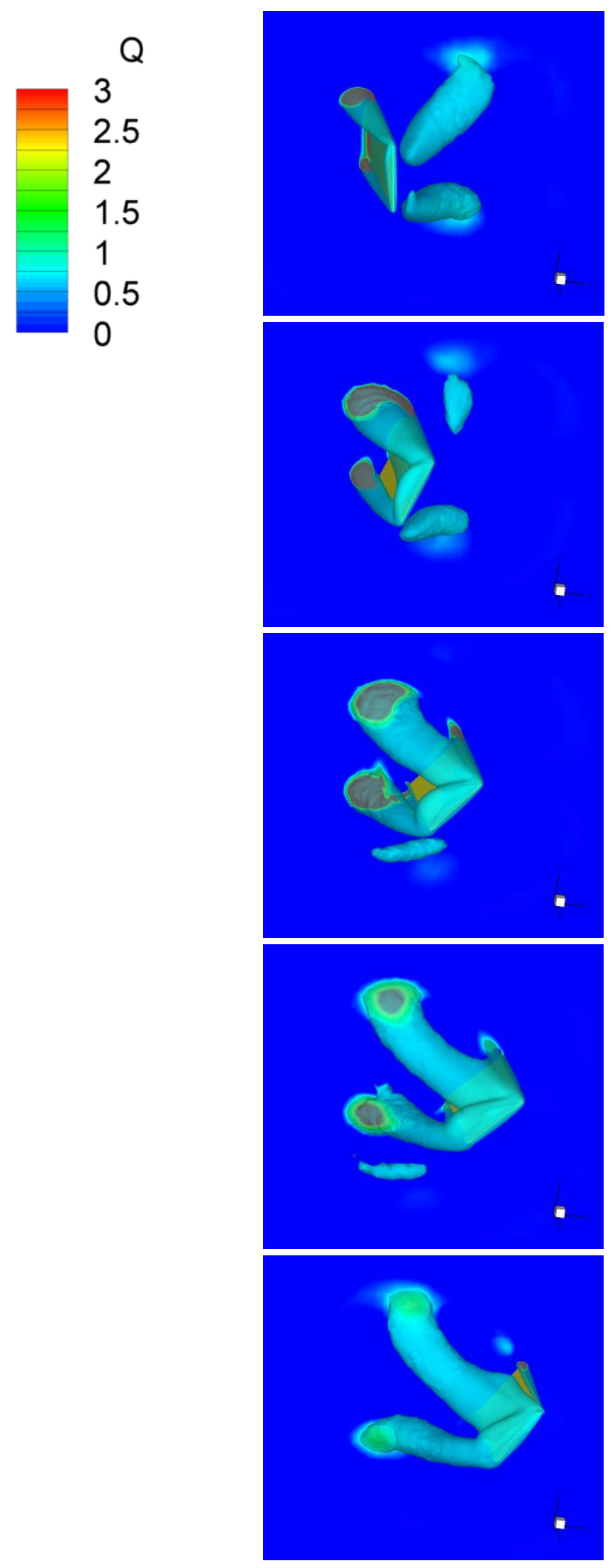

(a) iso-Q surface (0.75), the symmetry plane shows the Q contours Figure 19. Spanwise flow features for case $1\left(h_{a}=2.0, \alpha_{a}=45^{\circ}, \phi=60^{\circ}\right)$ at $t / T=0.8,0.9,1.0,1.1$, and 1.2. (a) iso-Q surface; (b) spanwise lift distribution from pressure.
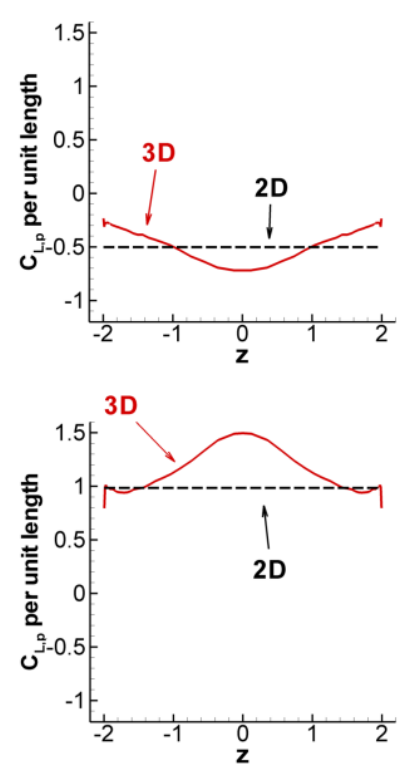

0.9
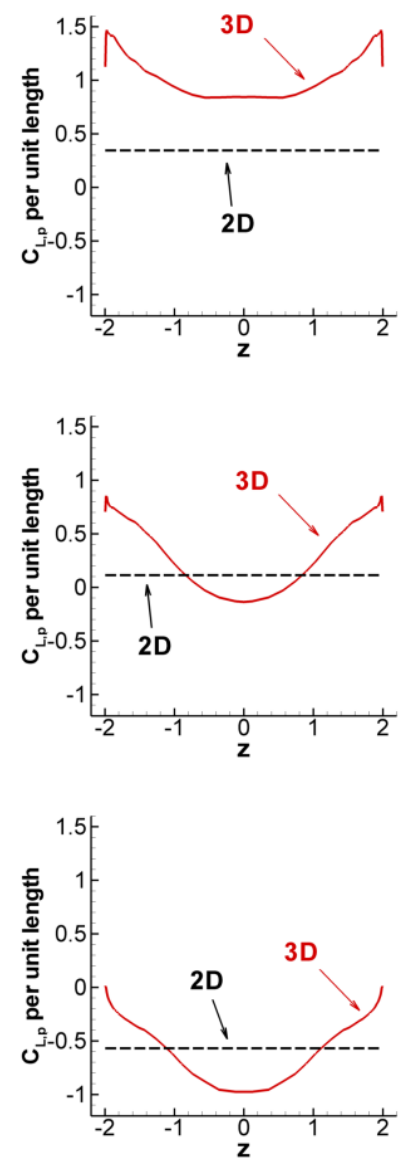

1.2

$\mathrm{t} / \mathrm{T}$ 
Vertical Velocity: $\quad-0.8-0.6-0.4 \quad-0.2 \quad 0 \quad 0.2 \quad 0.4 \quad 0.6 \quad 0.8$
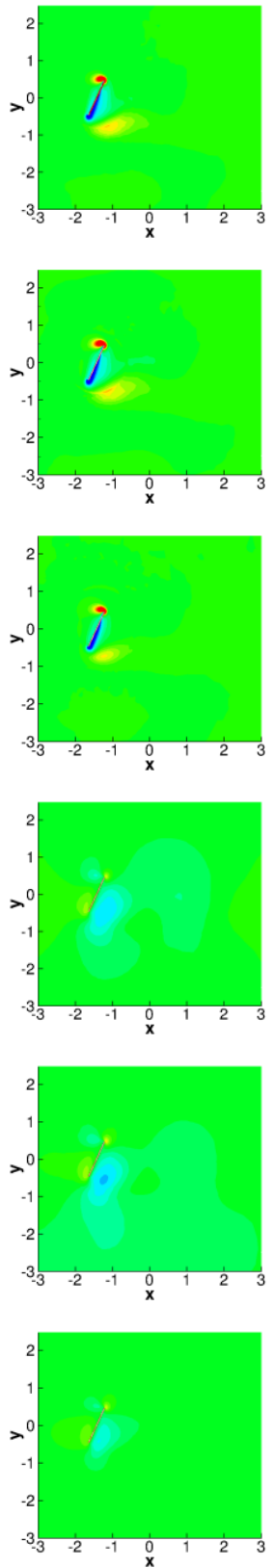

0.8
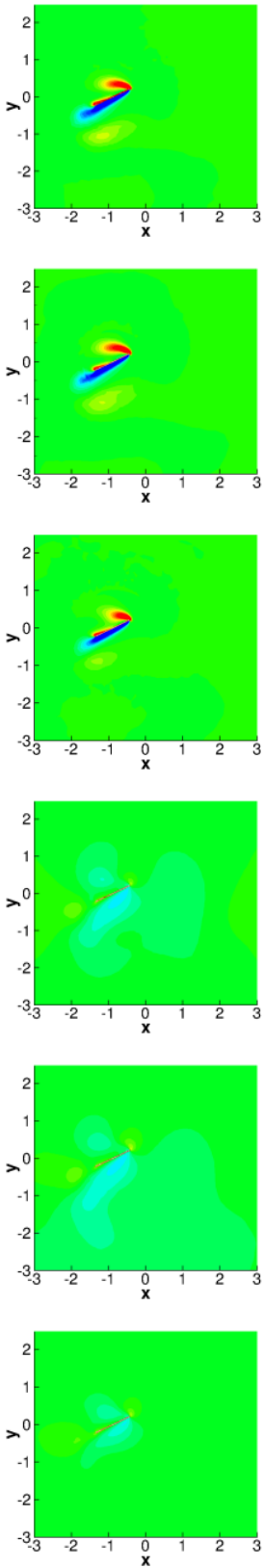

0.9
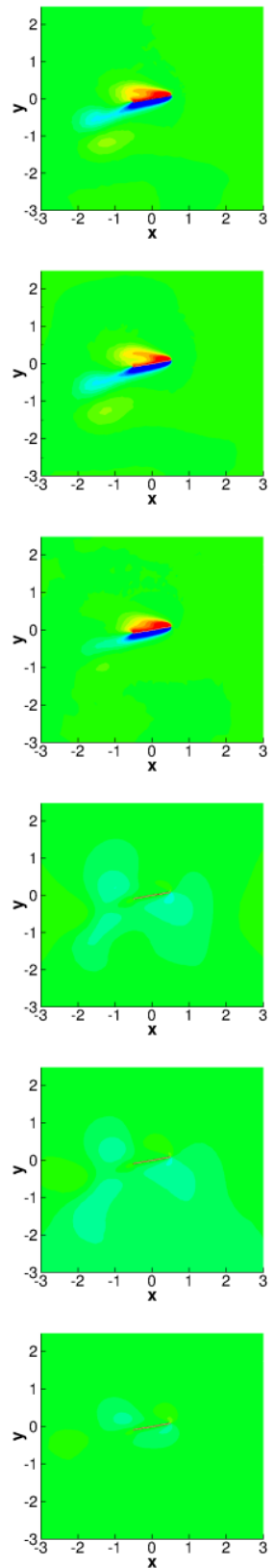

1.0

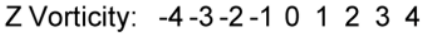
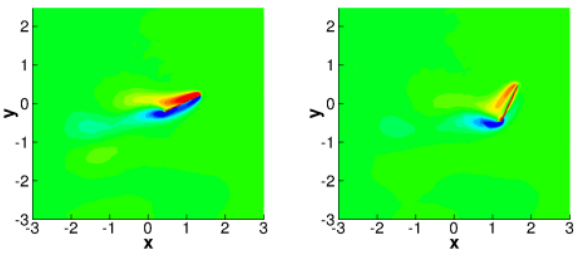

(a)
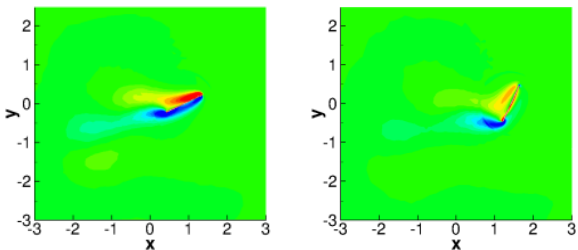

(b)
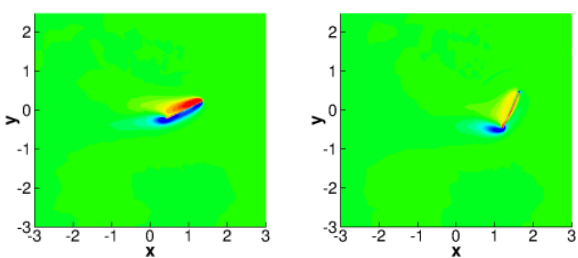

(c)
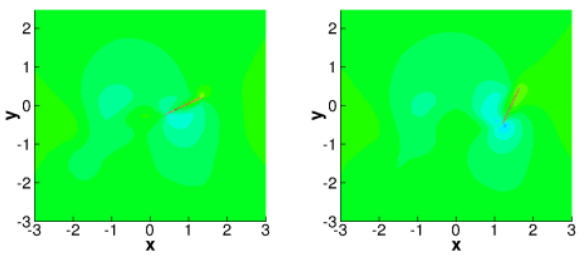

(d)
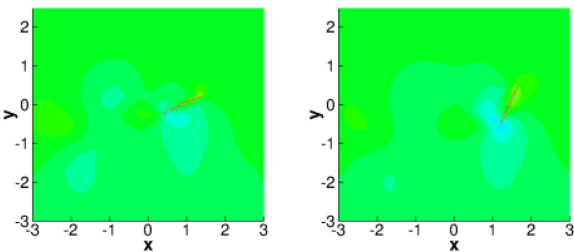

(e)
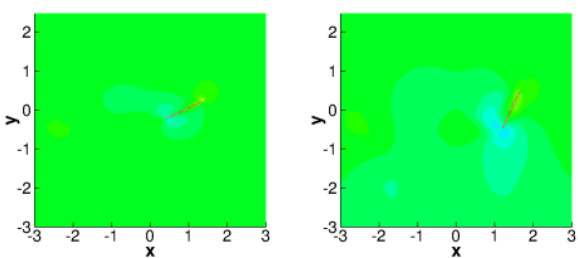

(f)

1.1

1.2

Figure 20. Contour plots at five time instants in the forward stroke, i.e. $t / T=0.8,0.9,1.0,1.1$, and 1.2 , for the case 12 $\left(h_{a}=3.0, \alpha_{a}=80^{\circ}, \phi=90^{\circ}\right)$ from 3D and 2D computations: (a) z-vorticity contours from 2D computation; (b) zvorticity contours in the symmetry plane of 3D computation; (c) z-vorticity contours near the wingtip $(\mathrm{z} / \mathrm{c}=1.8)$ plane from the 3D computation; (d) vertical velocity contours from $2 \mathrm{D}$ computation; (e) vertical velocity contours in the symmetry plane of $3 \mathrm{D}$ computation; (f) vertical velocity contours near the wingtip $(\mathrm{z} / \mathrm{c}=1.8)$ plane from the $3 \mathrm{D}$ computation. 


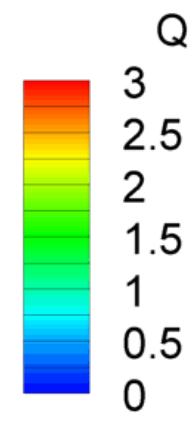

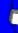
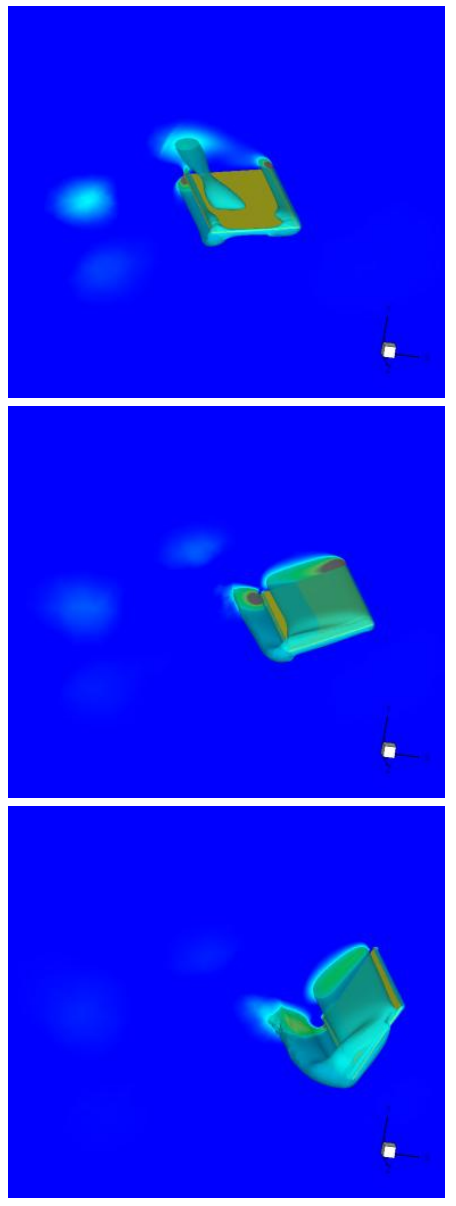

(a) iso-Q surface (0.75), the symmetry plane shows the Q contours Figure 21. Spanwise flow features for case $12\left(h_{a}=3.0, \alpha_{a}=80^{\circ}, \phi=90^{\circ}\right)$ at $t / T=0.8,0.9,1.0,1.1$, and 1.2. (a) iso-Q surface; (b) spanwise lift distribution from pressure.
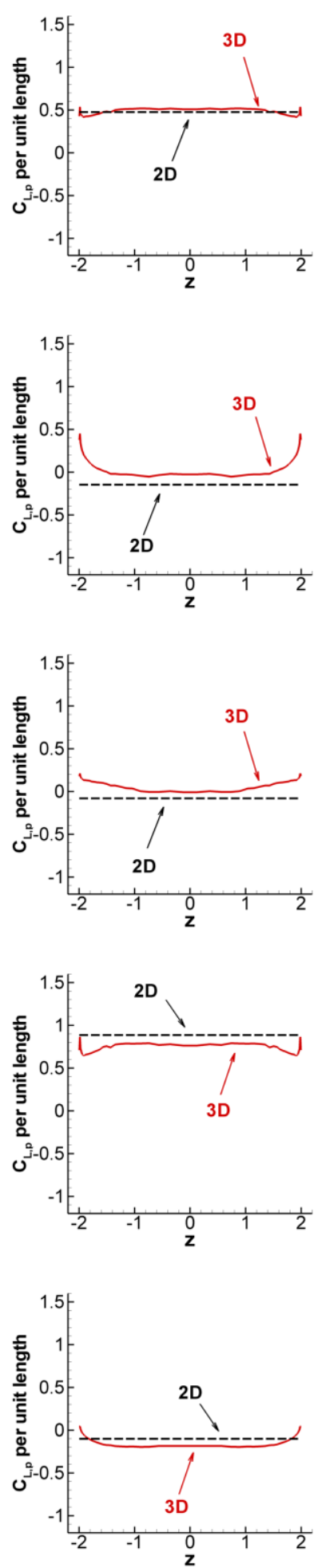
Case 12 - Similar $2 D$ and $3 D$ time history of $C_{L}$

Figure 20 shows the z-vorticity and the vertical velocity contours in the 2D flat plate computation, and 3D flat plate results in the symmetry plane, and near the wingtip $(\mathrm{z} / \mathrm{c}=1.8)$, respectively for $\mathrm{t} / \mathrm{T}=0.8,0.9,1.0,1.1$, and 1.2 for case 12. Contrasting the substantial spanwise variation in case 1 , in this case the difference among the two spanwise planes $(\mathrm{z} / \mathrm{c}=0.0,1.8)$ and the $2 \mathrm{D}$ flat plate computation is very small. The vorticity generation and the jet formed in previous strokes are also smaller than in case 1. As shown in Figure 21 the tip vortex distribution is smaller than in case 1 , because the angular amplitude for this case is $80^{\circ}$, leading to small angle of attack during the midstroke where the translational velocity is large. Figure 21(b) shows the spanwise lift distribution where the spanwise gradient vanishes except near the wingtips where we see some influence from the tip-vortex.

\section{Summary and Conclusion}

In this study we examined hovering kinematics of a $2 \mathrm{D}$ ellipse and $3 \mathrm{D}$ flat plate $(\mathrm{AR}=4)$ with the aid of surrogate models at a Reynolds number of 100. Regarding the fluid physics and aerodynamic implications, we observed:

(i) The role of the leading edge vortex and associated delayed stall played a dominant role in the determination of lift in both 2D and 3D cases. During delayed rotation the starting vortices detached causing a weaker LEV during midstroke. In 3D, the tip vortices during delayed rotation tended to repel the starting vortices at the leading and trailing edges, thus leading to a substantial variation in the spanwise flow. However, during advanced rotation, the starting vortex remained attached with no distinction between the starting vortex and the LEV, and enhances lift. Angle of attack, angular velocity, translational velocity, and translational acceleration all impact the formation and evolution of the LEV.

(ii) The manifestation of the wake capturing mechanism in 3D has been seen to change as the behavior of the shed vortices may differ between $2 \mathrm{D}$ and $3 \mathrm{D}$ cases. This is illustrated by one examined where the shed $2 \mathrm{D}$ vortices would remain in the plane of the plunging motion. The $3 \mathrm{D}$ counterparts are shed at angles such that they leave plane of the plunging wing and do not collide with the wing upon its return. However, under this condition the leading edge vortex can grow faster and augment the lift.

(iii) In 2D, a persistent downward jet like flow feature was seen in to form in the wake sustained by the influence of shed vortices. In 3D at $\mathrm{Re}=100$, a weaker jet is encountered whose strength along the span varies.

(iv) There was significant variance in the spanwise distruibution of forces in the 3D. Cases which suppressed the unsteady mechanisms, those with the highest angular amplitudes and thus low angles of attack, appeared to have a relatively constant response along the span. In contrast 3D cases with a prominent tip vortices exhibited significant variations along the span which did not have a strong correlation to the $2 \mathrm{D}$ values experienced.

(v) At low Reynolds numbers the 3D flow physics and tip effects can promote lift due to a weakened jet interaction and more stable LEV near the tip region.

The surrogate modeling techniques revealed that:

(i) The weighted average surrogate agreed remarkably well at the independent test points. While the number of independent points was limited, the error was often less than a few percent. The maximum error that was found was less than $15 \%$.

(ii) The hierarchy of variable sensitivity in the time averaged lift changed between 2D and 3D. In 2D the importance was 1) angular amplitude 2) phase lag and 3) plunging amplitude. In 3D the hierarchy switches to 1) phase lag 2) angular amplitude and 3) plunging amplitude. This is in large part from the 3D simulations not suffering as large a penalty from the lift valley that would occur between wake capture and delayed stall. This difference in 2D and 3D is partially attributed to the absence of the jet interaction in 3D simulations.

(iii) The predictions of the power required remained largely the same between $2 \mathrm{D}$ and $3 \mathrm{D}$ design spaces. This implies that the unsteady effects which influence the drag can in some contexts be reduced down to their $2 \mathrm{D}$ counterparts. 

that all optimal conditions in 3D were better than their 2D counterparts. Furthermore many non-optimal points in 3D, those not on the Pareto front, outperformed the optimal 2D Pareto front.

The surrogates modeling techniques provided a useful method for approximating otherwise computationally expensive simulations without sacrificing fidelity. They proved an efficient method for analyzing trends seen throughout the design space and possible points of interest. Coupled with instantaneous force histories and flowfield measurements they can provide insight to the complex interplay of the physical mechanisms involved. We will investigate the effect of the Reynolds number and structural flexibility in the future.

\section{Appendix}

\section{A. 2D Ellipse Spatial and Temporal Sensitivity Study}

The spatial and temporal sensitivity studies are shown in Ref. 32. As can be seen in Figure 22, 2D ellipse grid convergence is attained at resolutions of $161 \times 161$ and higher. The converged results shown here are broadly consistent with those obtained using another computational algorithm, based on higher-order finite difference schemes $^{72,73}$, as seen in Figure 23. Because of the computational cost involved in running all of the simulations as well as the expected level of accuracy output by the surrogate models, the error encountered with the $81 \times 81$ grids was deemed acceptable for the 2D ellipse simulations. This is put in the context of the PRESS calculations,see Appendix B, and independent testing points which suggest that errors were less than $10 \%$. Based on the temporal sensitivity study a timestep of T/1000 was chosen because it provided the best accuracy and simulation walltime combination (note: $\mathrm{dt}=\mathrm{T} / 500$ computations required more walltime to reach comparable solutions at $\mathrm{dt}=\mathrm{T} / 1000$ ).
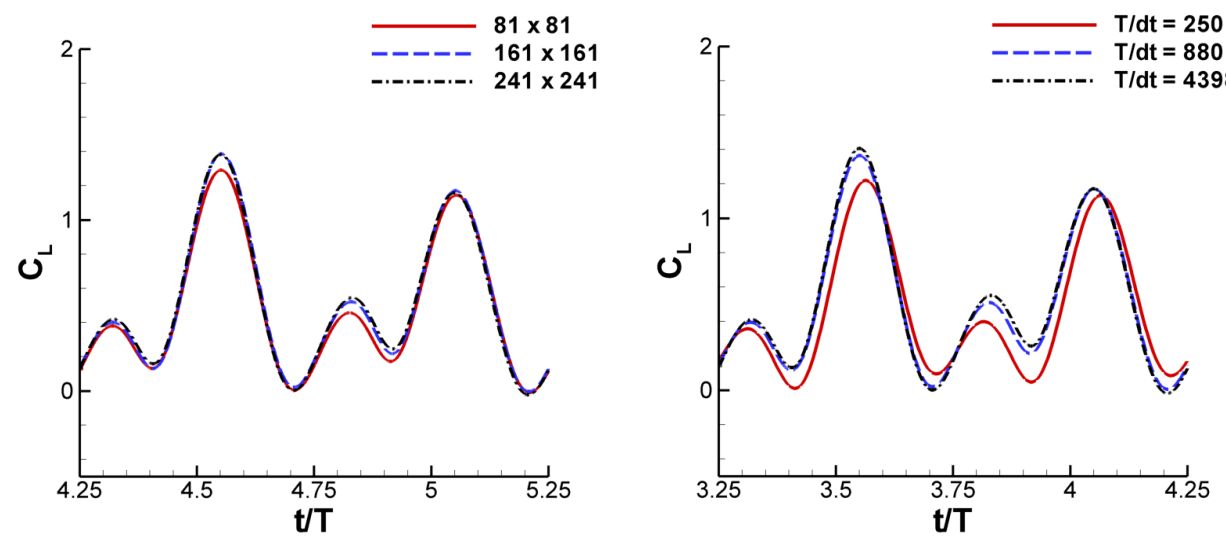

Figure 22. (Left): 2D ellipse grid sensitivity for normal hovering at $\operatorname{Re}=75,2 \mathrm{ha} / \mathrm{c}=2.8, \alpha a=45^{\circ}, \varphi=90^{\circ}$. (Right): $2 \mathrm{D}$ ellipse temporal sensitivity for normal hovering at $\operatorname{Re}=75,2 \mathrm{~h}_{\mathrm{a}} / \mathrm{c}=2.8, \alpha_{\mathrm{a}}=45^{\circ}, \varphi=90^{\circ}$.

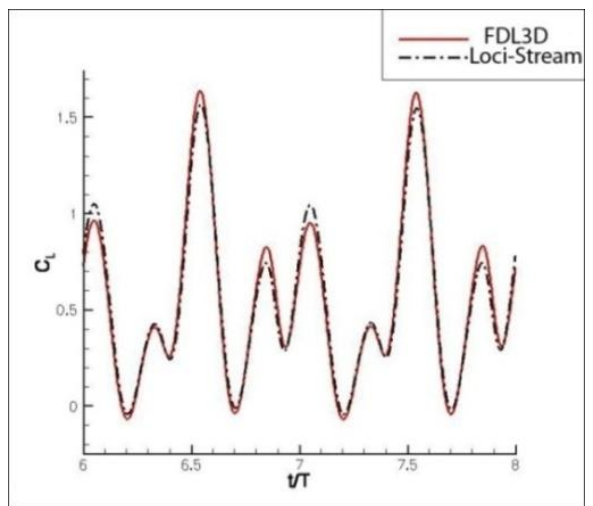

Figure 23. Cross-validation of Loci-Stream and FDL3DI for a 2D ellipse normal hovering computation with for Re $=100$, $2 \mathrm{ha} / \mathrm{c}=3.0, \alpha_{\mathrm{a}}=45^{\circ}, \varphi=90^{\circ}$. 


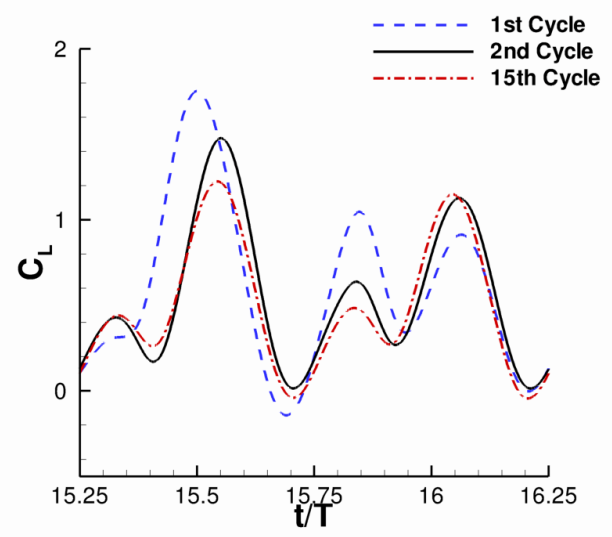

Figure 24. Illustration of the magnitude of the jet influence on 2D elliptic airfoil performance. The jet's structure has not reached a stable configuration during the first few cycles and therefore, most of the discrepancy can be attributed to this unsteady flow feature.

As can be verified in Figure 24, the 2D ellipse force histories do not immediately assume repeatable amplitudes. The changes between cycles had largely plateaued by the $15^{\text {th }}$ cycle for the $81 \times 81$ grid resolution, and accordingly the time averaged values for cases without freestream were taken over cycles 15 through 20.

\section{B. Case descriptions}

\begin{tabular}{|c|c|c|c|c|c|c|c|}
\hline$\alpha_{0}=90^{\circ}$ & $2 h_{a} / c$ & $\alpha_{a}$ & $\varphi$ & $\begin{array}{c}2 D \\
R e=100 \\
\left\langle C_{L}\right\rangle\end{array}$ & $\begin{array}{c}2 D \\
R e=100 \\
\left\langle P_{r e q}\right\rangle\end{array}$ & $\begin{array}{c}3 D \\
R e=100 \\
\left\langle C_{L}\right\rangle\end{array}$ & $\begin{array}{c}3 D \\
R e=100 \\
\left\langle P_{r e q}\right\rangle\end{array}$ \\
\hline Case 1 & 2.0 & $45^{\circ}$ & $60^{\circ}$ & 0.07 & 0.79 & 0.22 & 1.07 \\
\hline Case 2 & 2.0 & $45^{\circ}$ & $120^{\circ}$ & 0.51 & 1.06 & 0.75 & 1.02 \\
\hline Case 3 & 2.0 & $80^{\circ}$ & $60^{\circ}$ & 0.05 & 0.86 & -0.19 & 0.66 \\
\hline Case 4 & 2.0 & $80^{\circ}$ & $120^{\circ}$ & -0.05 & 0.63 & 0.30 & 0.66 \\
\hline Case 5 & 4.0 & $45^{\circ}$ & $60^{\circ}$ & 0.20 & 0.74 & 0.27 & 0.86 \\
\hline Case 6 & 4.0 & $45^{\circ}$ & $120^{\circ}$ & 0.62 & 0.89 & 0.70 & 0.83 \\
\hline Case 7 & 4.0 & $80^{\circ}$ & $60^{\circ}$ & -0.02 & 0.56 & 0.01 & 0.49 \\
\hline Case 8 & 4.0 & $80^{\circ}$ & $120^{\circ}$ & 0.08 & 0.47 & 0.32 & 0.47 \\
\hline Case 9 & 2.0 & $62.5^{\circ}$ & $90^{\circ}$ & 0.18 & 0.44 & 0.34 & 0.43 \\
\hline Case 10 & 4.0 & $62.5^{\circ}$ & $90^{\circ}$ & 0.34 & 0.44 & 0.42 & 0.42 \\
\hline Case 11 & 3.0 & $45^{\circ}$ & $90^{\circ}$ & 0.50 & 0.70 & 0.54 & 0.71 \\
\hline Case 12 & 3.0 & $80^{\circ}$ & $90^{\circ}$ & 0.07 & 0.30 & 0.17 & 0.28 \\
\hline Case 13 & 3.0 & $62.5^{\circ}$ & $60^{\circ}$ & 0.02 & 0.65 & 0.11 & 0.68 \\
\hline Case 14 & 3.0 & $62.5^{\circ}$ & $120^{\circ}$ & 0.36 & 0.73 & 0.58 & 0.68 \\
\hline Case 15 & 3.0 & $62.5^{\circ}$ & $90^{\circ}$ & 0.28 & 0.44 & 0.38 & 0.42 \\
\hline Case 16 & 3.6 & $66^{\circ}$ & $78^{\circ}$ & 0.21 & 0.40 & 0.29 & 0.41 \\
\hline Case 17 & 2.9 & $47^{\circ}$ & $91^{\circ}$ & 0.48 & 0.67 & 0.55 & 0.67 \\
\hline Case 18 & 3.8 & $69^{\circ}$ & $115^{\circ}$ & 0.27 & 0.55 & 0.48 & 0.51 \\
\hline Case 19 & 2.0 & $52^{\circ}$ & $67^{\circ}$ & 0.11 & 0.66 & $\mathrm{xxx}$ & $\mathrm{xxx}$ \\
\hline Case 20 & 2.8 & $77^{\circ}$ & $98^{\circ}$ & 0.07 & 0.34 & 0.24 & 0.33 \\
\hline Case 21 & 3.4 & $45^{\circ}$ & $120^{\circ}$ & $\mathrm{xxx}$ & $\mathrm{xxx}$ & 0.72 & 0.87 \\
\hline Case 22 & 4.0 & $45^{\circ}$ & $90^{\circ}$ & 0.51 & 0.70 & 0.54 & 0.68 \\
\hline Case 23 & 4.0 & $62.5^{\circ}$ & $120^{\circ}$ & 0.42 & 0.68 & 0.59 & 0.62 \\
\hline Case 24 & 3.0 & $80^{\circ}$ & $120^{\circ}$ & 0.02 & 0.52 & 0.32 & 0.53 \\
\hline Case 25 & 3.0 & $45^{\circ}$ & $60^{\circ}$ & 0.18 & 0.72 & 0.25 & 0.92 \\
\hline Case 26 & 2.8 & $50^{\circ}$ & $70^{\circ}$ & $\mathrm{xxx}$ & $\mathrm{xxx}$ & 0.32 & 0.72 \\
\hline Case 27 & 2.6 & $55^{\circ}$ & $110^{\circ}$ & $\mathrm{xxx}$ & $\mathrm{xxx}$ & 0.61 & 0.68 \\
\hline
\end{tabular}


C. Surrogate Model Characteristics

\begin{tabular}{|l|l|l|}
\hline Model & Comment 1 & Comment 2 \\
\hline Kriging & Linear Regression Model & Gaussian Correlation Model \\
\hline PRS & $2^{\text {nd }}$ Order Polynomial & ---- \\
\hline RBNN & Max Neurons = 1000 & ----- \\
\hline SVR1 & Linear Spline Kernel & $\begin{array}{l}\text { Full- infinity as upper bound } \\
\text { (non-separable case) }\end{array}$ \\
\hline SVR2 & Linear Spline Kernel & Short- finite upper bound \\
\hline SVR3 & Exponential Kernel & Full \\
\hline SVR4 & Exponential Kernel & Short \\
\hline SVR5 & Gaussian Kernel & Full \\
\hline SVR6 & Gaussian Kernel & Short \\
\hline
\end{tabular}

\section{Predicted Values at Independent Test Points}

\begin{tabular}{|l|c|c|c|c|l|c|l|l|l|}
\hline & $\mathbf{2 h}_{\boldsymbol{a}} / \boldsymbol{c}$ & $\boldsymbol{a}_{\boldsymbol{a}}$ & $\boldsymbol{\varphi}$ & 2D & 2D & 3D & 3D & 3D & 3D \\
\hline & & & & Lift (V) & Power (V) & Lift (V) & Power (V) & Lift (G) & Power (G) \\
\hline Case 22 & 4.0 & $45^{\circ}$ & $90^{\circ}$ & 0.54 & 0.67 & 0.53 & 0.66 & 0.53 & 0.66 \\
\hline Case 23 & 4.0 & $62.5^{\circ}$ & $120^{\circ}$ & 0.38 & 0.71 & 0.51 & 0.62 & 0.57 & 0.62 \\
\hline Case 24 & 3.0 & $80^{\circ}$ & $120^{\circ}$ & 0.02 & 0.53 & 0.33 & 0.59 & 0.33 & 0.58 \\
\hline Case 25 & 3.0 & $45^{\circ}$ & $60^{\circ}$ & 0.14 & 0.77 & 0.25 & 0.93 & 0.25 & 0.93 \\
\hline
\end{tabular}

\section{E. Definition of $\mathbf{Q}$}

To visualize the vortical structures the second invariant of the velocity gradient tensor, $Q$, has been used. $Q$ is defined as

$$
Q=-\frac{1}{2} \frac{\partial u_{i}}{\partial x_{j}} \frac{\partial u_{j}}{\partial x_{i}}=-\frac{1}{2}\left(S_{i j} S_{i j}-\frac{1}{2} \omega_{i} \omega_{i}\right)
$$

see Ref. 74 and measures the degree of vorticity, $\omega_{i}$ compared to the rate-of-strain, $S_{i j}$, from the local velocity gradients.

\section{Acknowledgments}

The work supported here has been supported in part by the Air Force Office of Scientific Research's Multidisciplinary University Research Initiative (MURI) grant and by the Michigan/AFRL (Air Force Research Laboratory)/Boeing Collaborative Center in Aeronautical Sciences. We thank Dr. Hikaru Aono for the fruitful discussions he provided.

\section{References}

${ }^{1}$ Shyy, W., Lian, Y., Tang, J., Viieru, D., and Liu, H., Aerodynamics of Low Reynolds Number Flyers, Cambridge University Press, New York, 2008.

${ }^{2}$ Mueller, T.J., and DeLaurier, J.D., “Aerodynamics of Small Vehicles,” Annual Review of Fluid Mechanics, Vol. 35, 2003 , pp. 89-111.

${ }^{3}$ Bohorquez, F., Samuel, P., Sirohi, J., Rudd, L, and Pines, D., "Design Analysis and Hover Performance of a Rotary Wing Micro Air Vehicle," Journal of American Helicopter Society, Vol. 48, No. 2, 2003, pp. 80-90.

${ }^{4}$ Shyy, W., Berg, M., and Ljungqvist, D., "Flapping and Flexible Wings for Biological and Micro Air Vehicles", Progress in Aerospace Sciences, Vol. 35, 1999, pp. 455-505.

${ }^{5}$ Fearing R.S., Chiang, K.H., Dickinson M., Pick, D.L, Sitti, M., and Yan, J., "Wing Transmission for a Micromechanical Flying Insect," IEEE International Conference on Robotics and Automation, San Francisco, CA, 2000. pp. 1509-1515.

${ }^{6}$ Lentink, D., Bradshaw, N., and Jongerius, S.R., "Novel Micro Aircraft Inspired by Insect Flight," Comparitive Biochemistry and Physiology A-Molecular \& Integrative Physiology, Vol. 146, No. 4, 2007, pp. 133-134. 
${ }^{7}$ Pornsin-Sirirak, T.N., Keenon, M., Lee, S.W., Tai, Y.C., Grasmeyer, J., Nassef, H., and Ho, C.M., "MEMS Wing Technology for a Battery-Powered Ornithopter," $13^{\text {th }}$ IEEE International Conference on Micro Electro Mechanical Systems, Miyazaki, Japan, January 23-27, 2000, pp. 799-804.

${ }^{8}$ Ellington, C.P., "Limitations on Animal Flight Performance," Journal of Experimental Biology, Vol. 160, 1991, pp. 71-91.

${ }^{9}$ Spedding, G.R., and Lissaman, P.B.S., "Technical Aspects of Microscale Flight Systems," Journal of Avian Biology, Vol. 29, No. 4, 1998, pp. 458-468.

${ }^{10}$ Ellington, C.P, "Novel Aerodynamics of Insect Flight: Applications to Micro-Air Vehicles," Jornal of Experimental Biology, Vol. 202, 1999, pp. 3439-3448.

${ }^{11}$ Norberg, U.M.L., "Structure, Form, and Function of Flight in Engineering and the Living World," Journal of Morphology, Vol. 252, No. 1, 2002, pp. 52-81.

${ }^{12}$ Rozhdestvensky, K.V. and Ryzhov, V.A., “Aerohydrodynamics of Flapping-Wing Propulsors," Progress in Aerospace Sciences, Vol. 39, 2003. pp. 585-633.

${ }^{13}$ Ho, S., Nassef, H., Pornsin-Sirirak, N., et al. "Unsteady Aerodynamics and Flow Control for Flapping Wing Flyers," Progress in Aerospace Sciences, Vol. 39, No. 8, 2003, pp. 635-681.

${ }^{14}$ Pines, D. and Bohorquez, "Challenges Facing Future Micro-Air-Vehicle Development," Journal of Aircraft, Vol. 43, No. 2, 2006, pp. 290-305.

${ }^{15}$ Wang, Z. J., "Dissecting Insect Flight,” Annual Review of Fluid Mechanics, Vol. 37, 2005, pp. 183-210.

${ }^{16}$ Ansari, S.A., Zbikowski, R., and Knowles, K., “Aerodynamic Modeling of Insect-like Flapping Flight for Micro Air Vehicles,” Progress in Aerospace Sciences, Vol. 42, 2006, pp. 129-172.

${ }^{17}$ Togashi, F., Ito, Y., Murayama, M., Nakahashi, K., Kato, T., "Flow Simulation of Flapping Wings of an Insect Using Overset Unstructured Grid," AIAA Paper 2001-2619, $15^{\text {th }}$ AIAA Computational Fluid Dynamics Conference, Anaheim, CA, June 11-14, 2001.

${ }^{18}$ Aono, H., Shyy, W., and Lui, H., "Vortex Dynamics in Near Wake of a Hovering Hawkmoth," AIAA Paper 2008-583, 46 ${ }^{\text {th }}$ AIAA Aerospace Sciences Meeting and Exhibit, Reno, NV, January 7-10, 2008.

${ }^{19}$ Shyy, W., and Liu, H., "Flapping Wings and Aerodynamic Lift: The Role of Leading-Edge Vortices," AIAA Journal, Vol. 45, No. 12, 2007

${ }^{20}$ Shyy, W., Lian, Y., Tang, J., Liu, H., Trizila, P., Stanford, B., Ifju, P., Bernal, L., Cesnik, C., and Friedmann, P., "Computational Aerodynamics of Low Reynolds Number Plunging, Pitching and Flexible Wings", Acta Mechanica Sinica, Vol. 24, No. 4, 2008, pp. 351-373.

${ }^{21}$ Warrick, D., Tobalske, B.W., and Powers, D.R., “Aerodynamics of the Hovering Hummingbird,” Nature, Vol. 435, 2005 , pp. 1094-1097.

${ }^{22}$ Sallstrom E., and Ukeiley, L., “Three Dimensional Averaged Flow Field Around Rigid Flapping Wings,” AIAA Paper 2008$3721, / 38^{\text {th }}$ AIAA Fluid Dynamics Conference and Exhibit, June 2008.

${ }^{23}$ Spedding, G.R., Hedenström, A., Rosén, M., "Quantitative Studies of the Wakes of Freely Flying Birds in a Low-Turbulence Wind Tunnel," Exp. Fluids, Vol. 34, 2003, pp. 291-303.

${ }^{24}$ Ellington, C.P., van den Berg, C., Willmott, A.P., and Thomas, A.L.R., "Leading-Edge Vortices in Insect Flight," Nature, Vol. 384, 1996, pp. 626-630.

${ }^{25}$ Fry, S., N., Sayaman, R., and Dickinson, M.H. "The Aerodynamics of Free-Flight Maneuvers in Drosophila," Science, Vol. 300, 2003, pp. 295-298.

${ }^{26}$ Bomphrey, R.J., Lawson, N.J., Harding, N.J., Taylor, G.K., and Thomas, A.L.R., "The Aerodynamics of Manduca Sexta: Digital Particle Image Velocimetry Analysis of the Leading-Edge Vortex," Journal of Experimental Biology, Vol. 208, 2005, pp. 1079-1094.

${ }^{27}$ Srylgley, R.B., and Thomas, A.L.R., "Unconventional Lift-Generating Mechanisms in Free-Flying Butterflies," Nature, Vol. 420, 2002, pp. 660-664.

${ }^{28}$ Wang, Z.J., and Russell, D., "The Effect of Fore and Hind-Wing Interactions on Aerodynamic Force and Power in Dragonfly Flight," Physical Review, Vol. 99, No. 14, 2007.

${ }^{29}$ Muijres, F.T., Johansson, L.C., Barfield, R., Wolf, M., Spedding, G.R., and Hedenstrom, A., "Leading-Edge Vortex Improves Lift in Slow-Flying Bats," Science, Vol. 319, 2008, pp. 1250-1253.

${ }^{30}$ Tolbalske, B.W., Warrick, D.R., Clark, C.J., Powers, D.R., Hedrick, T.L, Hyder, G.A., and Biewener, A., "Three-dimensional Kinematics of Hummingbird Flight," Journal of Experimental Biology, Vol. 210, 2007, pp. 2368-2382.

${ }^{31}$ Henningsoon, P., Spedding, G.R., and Hedenström, A., "Vortrex Wake and Flight Kinematics of a Swift in Cruising Flight in a Wind Tunnel," Journal of Experimental Biology, Vol. 211, 2008, pp. 717-730.

${ }^{32}$ Kesel,A.B., "Aerodynamic Characteristics of Dragonfly Wing Sections Compared with Technical Airfoils," Journal of Experimental Biology, Vol. 203, 2000, pp. 3125-3135.

${ }^{33}$ Tamai, M., Wang, Z., Rajagopalan, G., Hu, H, “Aerodynamic Performance of a Corrugated Dragonfly Airfoil Compared with Smooth Airfoils at Low Reynolds Numbers," AIAA Paper 2007-483, 45 ${ }^{\text {th }}$ AIAA Aerospace Sciences Meeting and Exhibit, Reno, NV, January 8-11, 2007.

${ }^{34}$ Isogai, K. and Shinmoto, Y., "Study on Aerodynamic Mechanism of Hovering Insects," AIAA Paper 2001-2470, 19 ${ }^{\text {th }}$ Applied Aerodynamics Conference, Anaheim, CA, June 11-14, 2001.

${ }^{35}$ Wang, Z.J., Birch, M. B., and Dickinson, M.H., "Unsteady Forces and Flows in Low Reynolds Number Hovering Flight: TwoDimensional Computatinos vs Robotic Wing Experiments," Journal of Experimental Biology, Vol. 207, 2004. pp. 461-474. 
${ }^{36}$ Kurtulus, D.F., David, L, Farcy, A., and Alemdaroglu, N., “Aerodynamic Characteristics of Flapping Motion in Hover," Experiments in Fluids, Vol. 44, 2008, pp. 23-36.

${ }^{37}$ Bos, F.M., Lentik, D., van Oudheusden, B.W., and Bijl, H., "Influence of Wing Kinematics on Aerodynamic Performance in Hovering Insect Flight,” Journal of Fluid Mechanics, Vol. 594, 2008, pp. 341-368.

${ }^{38}$ Trizila, P., Kang, C., Visbal, M.R., and Shyy, W., "Unsteady Fluid Physics and Surrogate Modeling of Low Reynolds Number, Flapping Airfoils", AIAA Paper No. 2008-3821, 38 ${ }^{\text {th }}$ Fluid Dynamics Conference and Exhibit, Seattle, WA, June 23-26, 2008.

${ }^{39}$ Sane, S.P. and Dickinson, M., "The Control of Flight Force by a Flapping Wing: Lift and Drag Production," Journal of Experimental Biology, Vol. 204, 2001, pp. 2607-2626.

${ }^{40}$ Weis-Fogh, T., "Quick Estimates of Flight Fitness in Hovering Animals, Including Novel Mechanisms for Lift Production," Journal of Experimental Biology., Vol. 59, 1973, pp. 169-230.

${ }^{41}$ Queipo, N., Haftka, R.T., Shyy, W., Goel, T. Vaidyanathan, R. and Tucker, P.K., "Surrogate-Based Analysis and Optimization," Progress in Aerospace Sciences, Vol. 41, 2005, pp. 1-25.

${ }^{42}$ Myers, R.H., and Montgomery, D.C., Response Surface Methodology: Process and Product Optimization Using Designed Experiments, Wiley, New York, 2002.

${ }^{43}$ Sacks, J., Schiller, S.B., and Welch, W.J., "Design for Computer Experiments," Technometrics, Vol. 31, No. 1, 1989 , pp. $41-47$.

${ }^{44}$ Cheng, B., and Titterington, D.M., "Neural Networks: a Review from a Statistical Perspective," Statistical Science, Vol. 9, 1994, pp. 2-54.

${ }^{45}$ Smola, A.J., and Scholkopf, B., “A Tutorial on Support Vector Regression,” Statistics and Computing, Vol. 14, 2004, pp.199222.

${ }^{46}$ Giunta, A.A., "Use of Data Sampling, Surrogate Models, and Numerical Optimization in Engineering Design," AIAA Paper No. 2002-538, 40 ${ }^{\text {th }}$ AIAA Aerospace Sciences Meeting and Exhibit, Reno, NV, Jan 14-17, 2002.

${ }^{47}$ Shyy, W., Papila, N., Vaidyanathan, R. and Tucker, P.K., "Global Design Optimization for Aerodynamics and Rocket Propulsion Components," Progress in Aerospace Sciences, Vol. 37, 2001, pp. 59-118.

${ }^{48}$ Eldred, M.S. and Dunlavy, D.M., "Formulations for Surrogate-Based Optimization with Data Fit, Multifidelity, and ReducedOrder Models," AIAA Paper No. 2006-7117, $11^{\text {th }}$ AIAA/ISSMO Multidisciplinary Analysis and Optimization Conference, Portsmouth, VA, Sept. 6-8, 2006.

${ }^{49}$ Glaz, B., Friedmann, P., and Liu, L., "Surrogate Based Optimization of Helicopter Rotor Blades for Vibration Reduction in Forward Flight," Structural and Multidisciplinary Optimization, Vol. 35, No. 4, 2008, pp. 341-363.

${ }^{50}$ Kanazaki, M., Tanaka, K., Shinkyu, J., and Yamamoto, K., "Multi-Objective Aerodynamic Exploration of Elements' Setting for High-Lift Airfoil Using Kriging Model," Journal of Aircraft, Vol. 44, No. 3, 2007, pp. 858-864.

${ }^{51}$ Madsen, J.I., Shyy, W. and Haftka, R.T., "Response Surface Techniques for Diffuser Shape Optimization," AIAA Journal, Vol. 38, 2000, pp. 1512-1518.

${ }^{52}$ Mack, Y., Goel, T., Shyy, W., and Haftka, R.T., "Multiple Surrogates for the Shape Optimization of Bluff Body-Facilitated Mixing," AIAA Paper No. 2005-333, 43 ${ }^{\text {rd }}$ AIAA Aerospace Sciences Meeting and Exhibit, Jan. 10-13, Reno, NV, 2005.

${ }^{53}$ Goel, T., Dorney, D.J., Haftka, R.T., and Shyy, W., "Improving the Hydrodynamic Performance of Diffuser Vanes via Shape Optimization," Computer \& Fluids, Vol. 37, No. 6, 2008, pp. 705-723.

${ }^{54}$ Samad, A., Kim, K., Goel, T., Haftka, R.T., and Shyy, W., "Multiple Surrogate Modeling for Axial Compressor Blade Shape Optimization," Journal of Propulsion and Power, Vol. 24, No. 2, 2008, pp. 302-310.

${ }^{55}$ Meaders, J. and Mattson, C., "Robust Design Optimization of a Constant Force Mechanism using a Surrogate Modeling Approach," AIAA Paper No. 2008-900, 46 ${ }^{\text {th }}$ AIAA Aerospace Sciences Meeting and Exhibit, Reno, NV, Jan. 7-10, 2008.

${ }^{56}$ Madsen, J.I., Shyy, W. and Haftka, R.T., "Response Surface Techniques for Diffuser Shape Optimization," AIAA Journal, Vol. 38, 2000, pp. 1512-1518.

${ }^{57}$ Cho, Y., Jayaraman, B., Viana, F.A.C., Haftka, R.T., Shyy, W., "Surrogate Modeling for Characterizing the Performance of Dielectric Barrier Discharge Plasma Actuator," AIAA Paper No. 2008-1381, $46^{\text {th }}$ AIAA Aerospace Sciences Meeting and Exhibit, Reno, NV, Jan. 7-10, 2008.

${ }^{58}$ Zhang, X., Sastry, A.M., and Shyy, W., "Intercalation-Induced Stress and Heat Generation within Single Lithium-Ion Battery Cathode Particles," Journal of The Electrochemical Society, Vol. 155, No. 5, 2008, pp. 542-552.

${ }^{59}$ Goel, T., Haftka, R.T., and Shyy, W., "Error Measures for Noise-free Surrogate Approximations," AIAA-Paper 2008-901, 46 ${ }^{\text {th }}$ AIAA Aerospace Sciences Meeting and Exhibit, Reno, NV, Jan. 7-10, 2008.

${ }^{60}$ Viana, F.A.C., Haftka, R.T., Valder, S., Butkewitsch, S, and Leal, M., "Ensemble of Surrogates: a Framework based on Minimization of the Mean Integrated Square Error," AIAA Paper No. 2008-1885, $49^{\text {th }}$ AIAA/ASME/ASCE/AHS/ASC Structures, Structural Dynamics, and Materials Conference, Shaumburg, IL, April 7-10, 2008.

${ }^{61}$ Goel, T., Haftka, R.T., Shyy, W., and Queipo, N.V., "Ensemble of Surrogates," Structural and Multidisciplinary Optimization, Vol. 33, No. 3, 2007.

${ }^{62}$ Luke, E.A., and George, T., "Loci: A Rule-Based Framework for Parallel Multi-Disciplinary Simulation Synthesis", Journal of Functional Programming, Vol. 15, No. 3, 2005, pp 477-502.

${ }^{63}$ Kamakoti, R., Thakur, S., Wright, J., and Shyy, W., "Validation of a new parallel all-speed CFD code in a rule-based framework for multidisciplinary applications", AIAA paper 2006-3063, June 2006.

${ }^{64}$ Shyy, W., " A Study of Finite Difference Approximations to Steady-State, Convection-Dominated Flow Problems", Journal of Computational Physics, Vol. 57, No. 3, 1985, pp. 415-438.

${ }^{65}$ Shyy, W., Computational Modeling for Fluid Flow and Interfacial Transport, Elsevier, Amsterdam, 1994.

${ }^{66}$ Blazek, J., Computational Fluid Dynamics: Principles and Applications, Elsevier, Amsterdam, 2001. 
${ }^{67}$ Thakur,S.T., and Wright, J., "Loci-STREAM: All-Speed CFD Solver for Arbitrary Polygonal Meshes in the Loci Framework", Streamline Numerics Inc., Gainesville, Florida, 2005(unpublished).

${ }^{68}$ Thomas, P.D., and Lombard, K., "The Geometric Conservation Law - A Link between Finite-Difference and Finite-Volume Methods of Flow Computation on Moving Grids", AIAA paper 1978-1208, July 1978.

${ }^{69}$ Kamakoti, R., and Shyy, W., "Evaluation of Geometric Conservation Law using Pressure-Based Fluid Solver and Moving Grid Technique", International Journal of Numerical Methods for Heat \& Fluid Flow, Vol. 14, No. 7, p. 851-865.

${ }^{70}$ Weis-Fogh, T., "Energentics of Flight in Hummingbirds and in Drosophila," Journal of Experimental Biology, Vol. 56, pp. 79104.

${ }^{71}$ Ellington, C.P., “The Aerodynamics of Hovering Insect Flight. III. Kinematics," Philosophical Transactions of the Royal Society of London, Series B, Vol. 305, pp. 41-78.

${ }^{72}$ Visbal, M.R., and Gaitonde, D.V., "High-Order-Accurate Methods for Complex Unsteady Subsonic Flows," AIAA Journal, Vol. 37, No. 10, 1999, pp. 1231-1239.

${ }^{73}$ Visbal, M.R., and Gaitonde, D.V., "On the Use of Higher-Order Finite-Difference schemes on Curvilinear and Deforming Meshes," Journal of Computational Physics, Vol. 181, No. 1, 2002, pp. 155-185.

${ }^{74}$ Hunt, J.C.R., Wray, A.A., and Moin, P., "Eddies, Streams, and Convergence Zones in Turbulent Flows," Center for Turbulence Research, Report CTR-S88, 1988. 